\title{
A fast collocation method for a static bond-based linear peridynamic model
}

\author{
Xuhao Zhang $^{a}$ Hong Wang $^{b}$ \\ ${ }^{a}$ School of Mathematics, Shandong University, Jinan, Shandong 250100, China \\ ${ }^{b}$ Department of Mathematics, University of South Carolina, Columbia, South Carolina \\ 29208, USA (e-mail address: hwang@math.sc.edu, telephone: 803-777-4321. fax: \\ 803-777-6527)
}

\begin{abstract}
We develop a fast collocation method for a steady-state bond-based linear peridynamic model in two space dimensions. The method reduces the computational work from $O\left(N^{2}\right)$ per Krylov subspace iteration in a traditional collocation method to $O(N \log N)$ and the memory requirement from $O\left(N^{2}\right)$ in a collocation method to $O(N)$, where $N$ is the number of unknowns in the discrete system. Furthermore, the method reduces the computational work of evaluating and assembling the stiffness matrix, which often constitutes a major portion of the overall computational work, from $O\left(N^{2}\right)$ in a collocation method to $O(N)$. The significant reduction of the CPU times and storage in the fast collocation method is achieved by carefully exploring the structure of the stiffness matrix of the collocation method without any lossy compression involved. In other words, the fast method is evaluated in an equivalent but more efficient manner. Therefore, the fast method generates identical numerical solutions as the traditional collocation method does and naturally inherits the stability and convergence properties that were already proved for a traditional collocation method. Numerical results are presented to show the utility of the fast method.
\end{abstract}

Keywords: fast collocation method, nonlocal diffusion model, peridynamic model

\section{Introduction}

The classical theory of continuum solid mechanics assumes that all internal forces act effectively through a zero distance and yields mathematical

Preprint submitted to Computer Methods in Applied and Mechanical EngineeringJune 28, 2016

(C) 2016. This manuscript version is made available under the Elsevier user license http://www.elsevier.com/open-access/userlicense/1.0/ 
models that are expressed in terms of partial differential equations. These models have difficulties to describe problems with evolving discontinuities, due to its differentiability assumption on displacement fields. The peridynamic models [34, 37], as a reformulation of classical continuum solid mechanics, yields nonlocal mathematical formulations that are based on long-range interactions. Constitutive models in the peridynamics depend on finite deformation vectors, instead of deformation gradients in classical constitutive models. Consequently, the peridynamic models are natural for the representation of discontinuities in displacement fields and the description of cracks and their evolution in materials.

To date, the peridynamic theory and related nonlocal models have been successfully applied in many important applications, including failure and damage in composite laminates [19, 20, 25, 48], crack propagation and branching $[16,17,21]$, crack nucleation [39], phase transformations in solids [7], impact damage [2, 32, 36, 43], polycrystal fracture [14], crystal plasticity [40], damage in concrete [13], and geomaterial fragmentation [23].

Mathematically, the peridynamic models give rise to integro-differential equations with singular kernels defined on circular material horizons, where spatial integration is employed to compute the contribution of internal forces in a body to the material response. As the governing equations in peridynamics are continuum models, different numerical approximation techniques can be used to discretize these equations which differ in computational expense, memory requirements, and implementational effort as well as accuracy, convergence, and stability of the numerical solutions [11]. For example, collocation methods and meshfree methods apply directly to the strong form of the governing equations in the peridynamic models, and are relatively simple to implement $[29,30,31,36,42,47]$. On the other hand, finite element discretizations of the governing equations in the peridynamic models are based on Galerkin weak forms, and enjoy high convergence rates and are suitable for a wider range of singular kernels [5, 9, 25, 42, 46, 53]. However, finite element methods double the number of spatial dimensions that need to be discretized, which means a significantly increased computational work and memory requirement.

Numerical methods for the peridynamic models present new numerical difficulties that were not encountered in those for the classical continuum solid mechnical models: (i) The accuracy and convergence of the numerical methods for the peridynamic models depends heavily on the accurate evaluation of the singular integrals, which are usually defined on a disk, 
in the entries of the stiffness matrices. Consequently, different numerical quadratures have been developed to effectively evaluate these singular integrals, which often constitute a major portion of the overall computational work $[2,18,26,27,29,36,51]$. (ii) Because of their nonlocality, numerical methods for the peridynamic models usually generate dense stiffness matrices in which the bandwidths increase to infinity as the mesh size decreases to zero. Direct solvers are widely used in the peridynamic modeling, which have $O\left(N^{2}\right)$ memory requirement to store the stiffness matrix and $O\left(N^{3}\right)$ computations to find the numerical solutions.

In this paper we develop fast numerical methods for the peridyanmic models via an alternative approach. Namely, we do not intend to develop a more accurate numerical discretization or numerical quadrature for these models. Rather, we explore the structure of the stiffness matrices of existing numerical methods in order to significantly reduce the computational work to evaluate and assemble the stiffness matrix and to compute the numerical solutions, as well as the memory requirement to store the stiffness matrix. The idea applies to different numerical methods and numerical quadratures in the literature. For definiteness, in this paper we develop a fast collocation method for a static bond-based linear peridynamic model in two space dimensions .

The rest of the paper is organized as follows. In Section 2 we present a piecewise bilinear collocation method for model problem (1). In section 3 we analyze the structure of the stiffness matrix and based on the properties of the displacement vector and the stiffness matrix we derive an appropriate decomposition. In Section 4 we prove the appropriately decomposed submatrices of the stiffness matrix are block-Toeplitz-Toeplitz-block (BTTB). In section 5 we develop an efficient storage mechanism for the stiffness matrix that reduces the storage from $O\left(N^{2}\right)$ to $O(N)$ and a fast Krylov subspace iterative method that reduces each iteration from $O\left(N^{2}\right)$ computations to $O(N \log N)$ computations. In section 6 we carry out numerical experiments to show the utility of the fast method. In section 7 we draw concluding remarks. 


\section{A static bond-based linear peridynamic model and its colloca- tion discretization}

We consider the following static bond-based linear peridynamic model in two space dimensions $[34,35,37]$

$$
\begin{aligned}
\int_{B_{\delta}(\mathbf{x})} \mathbf{C}\left(\mathbf{x}^{\prime}, \mathbf{x}\right)\left(\mathbf{u}\left(\mathbf{x}^{\prime}\right)-\mathbf{u}(\mathbf{x})\right) d \mathbf{x}^{\prime} & =\mathbf{b}(\mathbf{x}), & & \mathbf{x} \in \Omega, \\
\mathbf{u}(\mathbf{x}) & =\mathbf{g}(\mathbf{x}), & & \mathbf{x} \in \Omega_{c} .
\end{aligned}
$$

Here $\mathbf{C}$ is the micromodulus tensor of the form

$$
\mathbf{C}\left(\mathbf{x}^{\prime}, \mathbf{x}\right)=\sigma\left(\left\|\mathbf{x}^{\prime}-\mathbf{x}\right\|\right)\left[\frac{\left(\mathbf{x}^{\prime}-\mathbf{x}\right)}{\left\|\mathbf{x}^{\prime}-\mathbf{x}\right\|} \otimes \frac{\left(\mathbf{x}^{\prime}-\mathbf{x}\right)}{\left\|\mathbf{x}^{\prime}-\mathbf{x}\right\|}\right] .
$$

In (1) and $(2), \mathbf{x}:=(x, y)^{T}, \Omega=\left(0, x_{R}\right) \times\left(0, y_{R}\right)$ is a rectangular domain, the material horizon $B_{\delta}(\mathbf{x})$ is assumed to be a closed disk of radius $\delta>0$ centered at $\mathbf{x}$, where $\delta$ is refered to as the peridynamic horizon. $\Omega_{c}$ denotes a boundary zone surrounding $\Omega$ of width $\delta . \sigma(\|\mathbf{x}\|)$ is the kernel function of the integral operator. $\mathbf{u}(\mathbf{x})=[v(\mathbf{x}), w(\mathbf{x})]^{T}$ represents the displacement of the material, $\mathbf{b}(\mathbf{x})=\left[b^{v}(\mathbf{x}), b^{w}(\mathbf{x})\right]^{T}$ represents the external force density, and $\mathbf{g}(\mathbf{x})=\left[g^{v}(\mathbf{x}), g^{w}(\mathbf{x})\right]^{T}$ is the prescribed nonlocal boundary data imposed on the domain $\Omega_{c}$.

Let the positive integers $N_{x}$ and $N_{y}$ denote the numbers of intervals in the discretized domain in the $x$ and $y$ directions, respectively. We define a spatial partition in $\bar{\Omega}=\left[0, x_{R}\right] \times\left[0, y_{R}\right]$ by $x_{i}:=i h_{x}$ for $i=0,1, \ldots, N_{x}$ and $y_{j}:=j h_{y}$ for $j=0,1, \ldots, N_{y}$, where $h_{x}:=x_{R} / N_{x}$ and $h_{y}:=y_{R} / N_{y}$ are the mesh sizes in the $x$ and $y$ directions. To handle the discretization of the boundary zone $\Omega_{c}$, we extend the partition to the nodes $\left(x_{i}, y_{j}\right)$ for $i=-K+1, \ldots,-1,0,1, \ldots, N_{x}-1, N_{x}, N_{x}+1, \ldots, N_{x}+K-1$ and $j=$ $-L+1, \ldots,-1,0,1, \ldots, N_{y}-1, N_{y}, N_{y}+1, \ldots, N_{y}+L-1$. Here

$$
K:=\left\lceil\delta / h_{x}\right\rceil, \quad L:=\left\lceil\delta / h_{y}\right\rceil
$$

are the ceilings of $\delta / h_{x}$ and $\delta / h_{y}$, respectively.

Let $\psi(\xi)=1-|\xi|$ for $\xi \in[-1,1]$ or zero otherwise. The two dimensional pyramid functions $\phi_{i j}(x, y)$ centered at $\left(x_{i}, y_{j}\right)$ can be expressed as

$$
\begin{gathered}
\phi_{i j}(x, y)=\psi\left(\frac{x-x_{i}}{h_{x}}\right) \psi\left(\frac{y-y_{j}}{h_{y}}\right), \quad(x, y) \in \Omega \cup \Omega_{c}, \\
-K+1 \leqslant i \leqslant N_{x}+K-1, \quad-L+1 \leqslant j \leqslant N_{y}+L-1 .
\end{gathered}
$$


Then the $x$-component $v(x, y)$ and the $y$-component $w(x, y)$ of the displacement can be expressed as

$$
\begin{aligned}
& v(x, y)=\sum_{i^{\prime}=-K+1}^{N_{x}+K-1} \sum_{j^{\prime}=-L+1}^{N_{y}+L-1} v_{i^{\prime}, j^{\prime}} \phi_{i^{\prime}, j^{\prime}}(x, y), \\
& w(x, y)=\sum_{i^{\prime}=-K-1}^{N_{y}+L-1} \sum_{j^{\prime}=-L+1} w_{i^{\prime}, j^{\prime}} \phi_{i^{\prime}, j^{\prime}}(x, y) .
\end{aligned}
$$

For $-K+1 \leq i^{\prime} \leq 0, N_{x} \leq i^{\prime} \leq N_{x}+K-1$ and $-L+1 \leq j^{\prime} \leq 0$, $N_{y} \leq j^{\prime} \leq N_{y}+L-1, v_{i^{\prime}, j^{\prime}}=g^{v}\left(x_{i^{\prime}}, y_{j^{\prime}}\right)$ and $w_{i^{\prime}, j^{\prime}}=g^{w}\left(x_{i^{\prime}}, y_{j^{\prime}}\right)$ are specified in (5). Hence, we insert (5) into (1) and enforce the resulting equations at the collocation points $\left(x_{i}, y_{j}\right)$ for $i=1,2, \ldots, N_{x}-1$ and $j=1,2, \ldots, N_{y}-1$ to obtain the following scheme

$$
\begin{aligned}
& \int_{B_{\delta}\left(x_{i}, y_{j}\right)} \frac{\sigma\left(\left\|\left(x^{\prime}-x_{i}, y^{\prime}-y_{j}\right)\right\|\right)}{\left(\left(x^{\prime}-x_{i}\right)^{2}+\left(y^{\prime}-y_{j}\right)^{2}\right)}\left\{\left(x^{\prime}-x_{i}\right)^{2}\left[v_{i, j}-\sum_{i^{\prime}=-K+1}^{N_{x}+K-1} \sum_{j^{\prime}=-L+1}^{N_{y}+L-1} v_{i^{\prime}, j^{\prime}} \phi_{i^{\prime}, j^{\prime}}\left(x^{\prime}, y^{\prime}\right)\right]\right. \\
& \left.+\left(x^{\prime}-x_{i}\right)\left(y^{\prime}-y_{j}\right)\left[w_{i, j}-\sum_{i^{\prime}=-K+1}^{N_{x}+K-1} \sum_{j^{\prime}=-L+1}^{N_{y}+L-1} w_{i^{\prime}, j^{\prime}} \phi_{i^{\prime}, j^{\prime}}\left(x^{\prime}, y^{\prime}\right)\right]\right\} d x^{\prime} d y^{\prime}=b^{v}\left(x_{i}, y_{j}\right), \\
& \int_{B_{\delta}\left(x_{i}, y_{j}\right)} \frac{\sigma\left(\left\|\left(x^{\prime}-x_{i}, y^{\prime}-y_{j}\right)\right\|\right)}{\left(\left(x^{\prime}-x_{i}\right)^{2}+\left(y^{\prime}-y_{j}\right)^{2}\right)}\left\{\left(y^{\prime}-y_{j}\right)^{2}\left[w_{i, j}-\sum_{i^{\prime}=-K+1}^{N_{x}+K-1} \sum_{j^{\prime}=-L+1}^{N_{y}+L-1} w_{i^{\prime}, j^{\prime}} \phi_{i^{\prime}, j^{\prime}}\left(x^{\prime}, y^{\prime}\right)\right]\right. \\
& \left.+\left(x^{\prime}-x_{i}\right)\left(y^{\prime}-y_{j}\right)\left[v_{i, j}-\sum_{i^{\prime}=-K+1}^{N_{x}+K-1} \sum_{j^{\prime}=-L+1}^{N_{y}+L-1} v_{i^{\prime}, j^{\prime}} \phi_{i^{\prime}, j^{\prime}}\left(x^{\prime}, y^{\prime}\right)\right]\right\} d x^{\prime} d y^{\prime}=b^{w}\left(x_{i}, y_{j}\right) .
\end{aligned}
$$

The collocation scheme (6) can be written in a matrix form

$$
\mathbf{A}_{2 N} \mathbf{u}_{2 N}=\mathbf{b}_{2 N} .
$$

Here $N:=\left(N_{x}-1\right)\left(N_{y}-1\right)$ refers to the number of grid points at which the values of $v$ and $w$ need to be computed. The $2 N$-dimensional unknown displacement vector $\mathbf{u}_{2 N}$ and the right-hand side loading vector $\mathbf{b}_{2 N}$ of (6) are labelled in their natural order. At each node $\left(x_{i}, y_{j}\right)$, the $x$ component 
$v_{i, j}$ or $b_{i, j}^{v}$ is put first and the $y$ component $w_{i, j}$ or $b_{i, j}^{w}$ second:

$$
\begin{aligned}
\mathbf{u}_{2 N}:= & {\left[v_{1,1}, w_{1,1}, \ldots, v_{N_{x}-1,1}, w_{N_{x}-1,1}, v_{1,2}, w_{1,2}, \ldots, v_{N_{x}-1,2}, w_{N_{x}-1,2},\right.} \\
& \left.\ldots, v_{1, N_{y}-1}, w_{1, N_{y}-1}, \ldots, v_{N_{x}-1, N_{y}-1}, w_{N_{x}-1, N_{y}-1}\right]^{T} \\
\mathbf{b}_{2 N}:= & {\left[b_{1,1}^{v}, b_{1,1}^{w}, \ldots, b_{N_{x}-1,1}^{v}, b_{N_{x}-1,1}^{w}, b_{1,2}^{v}, b_{1,2}^{w}, \ldots, b_{N_{x}-1,2}^{v}, b_{N_{x}-1,2}^{w}\right.} \\
& \left.\ldots, b_{1, N_{y}-1}^{v}, b_{1, N_{y}-1}^{w}, \ldots, b_{N_{x}-1, N_{y}-1}^{v}, b_{N_{x}-1, N_{y}-1}^{w}\right]^{T}
\end{aligned}
$$

The $2 N$-by- $2 N$ stiffness matrix $\mathbf{A}_{2 N}$ can be written as the following $N$-by- $N$ block matrix

$$
\mathbf{A}_{2 N}=\left(\begin{array}{ccccc}
\mathbf{A}_{1,1} & \mathbf{A}_{1,2} & \ldots & \mathbf{A}_{1, N-1} & \mathbf{A}_{1, N} \\
\mathbf{A}_{2,1} & \mathbf{A}_{2,2} & \ldots & \mathbf{A}_{2, N-1} & \mathbf{A}_{2, N} \\
\vdots & \vdots & \ddots & \vdots & \vdots \\
\mathbf{A}_{N-1,1} & \mathbf{A}_{N-1,2} & \ldots & \mathbf{A}_{N-1, N-1} & \mathbf{A}_{N-1, N} \\
\mathbf{A}_{N, 1} & \mathbf{A}_{N, 2} & \ldots & \mathbf{A}_{N, N-1} & \mathbf{A}_{N, N}
\end{array}\right)
$$

where each entry of $\mathbf{A}_{2 N}$ is a 2-by-2 matrix

$$
\mathbf{A}_{m, n}=\left(\begin{array}{cc}
A_{m, n}^{v, v} & A_{m, n}^{v, w} \\
A_{m, n}^{w, v} & A_{m, n}^{w, w}
\end{array}\right)
$$

with the entries in $\mathbf{A}_{m, n}$ being defined by

$$
\begin{aligned}
A_{m, n}^{v, v} & :=\int_{B_{\delta}\left(x_{i}, y_{j}\right)} \frac{\sigma\left(\left\|\left(x^{\prime}-x_{i}, y^{\prime}-y_{j}\right)\right\|\right)}{\left(\left(x^{\prime}-x_{i}\right)^{2}+\left(y^{\prime}-y_{j}\right)^{2}\right)}\left(x^{\prime}-x_{i}\right)^{2}\left(\delta_{m, n}-\phi_{i^{\prime}, j^{\prime}}\left(x^{\prime}, y^{\prime}\right)\right) d x^{\prime} d y^{\prime}, \\
A_{m, n}^{v, w}: & =\int_{B_{\delta}\left(x_{i}, y_{j}\right)} \frac{\sigma\left(\left\|\left(x^{\prime}-x_{i}, y^{\prime}-y_{j}\right)\right\|\right)}{\left(\left(x^{\prime}-x_{i}\right)^{2}+\left(y^{\prime}-y_{j}\right)^{2}\right)}\left(x^{\prime}-x_{i}\right)\left(y^{\prime}-y_{j}\right)\left(\delta_{m, n}-\phi_{i^{\prime}, j^{\prime}}\left(x^{\prime}, y^{\prime}\right)\right) d x^{\prime} d y^{\prime}, \\
A_{m, n}^{w, w}: & : \int_{B_{\delta}\left(x_{i}, y_{j}\right)} \frac{\sigma\left(\left\|\left(x^{\prime}-x_{i}, y^{\prime}-y_{j}\right)\right\|\right)}{\left(\left(x^{\prime}-x_{i}\right)^{2}+\left(y^{\prime}-y_{j}\right)^{2}\right)}\left(y^{\prime}-y_{j}\right)^{2}\left(\delta_{m, n}-\phi_{i^{\prime}, j^{\prime}}\left(x^{\prime}, y^{\prime}\right)\right) d x^{\prime} d y^{\prime} .
\end{aligned}
$$

Here $\delta_{m, n}=1$ for $m=n$ or 0 otherwise, and $A_{m, n}^{w, v}=A_{m, n}^{v, w}$. The global indices $m$ and $n$ are related to the indices $(i, j)$ and $\left(i^{\prime}, j^{\prime}\right)$ by

$$
\begin{aligned}
& m=(j-1) \times\left(N_{x}-1\right)+i, \quad 1 \leqslant i \leqslant N_{x}-1,1 \leqslant j \leqslant N_{y}-1, \\
& n=\left(j^{\prime}-1\right) \times\left(N_{x}-1\right)+i^{\prime}, \quad 1 \leqslant i^{\prime} \leqslant N_{x}-1,1 \leqslant j^{\prime} \leqslant N_{y}-1 .
\end{aligned}
$$


The entries of the right-hand side vector $\mathbf{b}_{2 N}$ are given by

$$
\begin{aligned}
& b_{i, j}^{v}=b^{v}\left(x_{i}, y_{j}\right)+\sum_{\substack{-K+1 \leq i^{\prime \prime} \leq 0 \\
N_{x} \leq i^{\prime \prime} \leq N_{x}+K-1}} \sum_{\substack{-L+1 \leq j^{\prime \prime} \leq 0 \\
N_{y} \leq j^{\prime \prime} \leq N_{y}+L-1}} \int_{B_{\delta}\left(x_{i}, y_{j}\right)} \frac{\sigma\left(\left\|\left(x^{\prime}-x_{i}, y^{\prime}-y_{j}\right)\right\|\right)}{\left(\left(x^{\prime}-x_{i}\right)^{2}+\left(y^{\prime}-y_{j}\right)^{2}\right)} \\
& \times\left(x^{\prime}-x_{i}\right)^{2} g^{v}\left(x_{i^{\prime \prime}}, y_{j^{\prime \prime}}\right) \phi_{i^{\prime \prime}, j^{\prime \prime}}\left(x^{\prime}, y^{\prime}\right) d x^{\prime} d y^{\prime} \\
& \begin{array}{l}
+\sum_{\substack{-K+1 \leq i^{\prime \prime} \leq 0 \\
N_{x} \leq i^{\prime \prime} \leq N_{x}+K-1\\
}} \sum_{\substack{-L+1 \leq j^{\prime \prime} \leq 0 \\
N_{y} \leq j^{\prime \prime} \leq N y+L-1}} \int_{B_{\delta}\left(x_{i}, y_{j}\right)} \frac{\sigma\left(\left\|\left(x^{\prime}-x_{i}, y^{\prime}-y_{j}\right)\right\|\right)}{\left(\left(x^{\prime}-x_{i}\right)^{2}+\left(y^{\prime}-y_{j}\right)^{2}\right)} \\
\quad \times\left(x^{\prime}-x_{i}\right)\left(y^{\prime}-y_{j}\right) g^{w}\left(x_{i^{\prime \prime}}, y_{j^{\prime \prime}}\right) \phi_{i^{\prime \prime}, j^{\prime \prime}}\left(x^{\prime}, y^{\prime}\right) d x^{\prime} d y^{\prime},
\end{array} \\
& b_{i, j}^{w}=b^{w}\left(x_{i}, y_{j}\right)+\sum_{\substack{-K+1 \leq i^{\prime \prime} \leq 0 \\
N_{x} \leq i^{\prime \prime} \leq N_{x}+K-1}} \sum_{\substack{-L+1 \leq j^{\prime \prime} \leq 0 \\
N_{y} \leq j^{\prime \prime} \leq N_{y}+L-1}} \int_{B_{\delta}\left(x_{i}, y_{j}\right)} \frac{\sigma\left(\left\|\left(x^{\prime}-x_{i}, y^{\prime}-y_{j}\right)\right\|\right)}{\left(\left(x^{\prime}-x_{i}\right)^{2}+\left(y^{\prime}-y_{j}\right)^{2}\right)} \\
& \times\left(x^{\prime}-x_{i}\right)\left(y^{\prime}-y_{j}\right) g^{v}\left(x_{i^{\prime \prime}}, y_{j^{\prime \prime}}\right) \phi_{i^{\prime \prime}, j^{\prime \prime}}\left(x^{\prime}, y^{\prime}\right) d x^{\prime} d y^{\prime} \\
& \begin{array}{l}
+\sum_{\substack{-K+1 \leq i^{\prime \prime} \leq 0 \\
N \leq \leq i^{\prime \prime} \leq N x+K-1}} \sum_{\substack{-L+1 \leq j^{\prime \prime} \leq 0 \\
N_{y} \leq j^{\prime \prime} \leq N y+L-1}} \int_{B_{\delta}\left(x_{i}, y_{j}\right)} \frac{\sigma\left(\left\|\left(x^{\prime}-x_{i}, y^{\prime}-y_{j}\right)\right\|\right)}{\left(\left(x^{\prime}-x_{i}\right)^{2}+\left(y^{\prime}-y_{j}\right)^{2}\right)} \\
\quad \times\left(y^{\prime}-y_{j}\right)^{2} g^{w}\left(x_{i^{\prime \prime}}, y_{j^{\prime \prime}}\right) \phi_{i^{\prime \prime}, j^{\prime \prime}}\left(x^{\prime}, y^{\prime}\right) d x^{\prime} d y^{\prime},
\end{array} \\
& 1 \leqslant i \leqslant N_{x}-1,1 \leqslant j \leqslant N_{y}-1 \text {. }
\end{aligned}
$$

The indices $\left(i^{\prime \prime}, j^{\prime \prime}\right)$ refer to the nodes in $\Omega_{c}$.

Because of the nonlocal property of the peridynamic model (1), the stiffness matrix $\mathbf{A}_{2 N}$ is dense since $K=O\left(\delta / h_{x}\right)$ and $L=O\left(\delta / h_{y}\right)$ tend to infinity as $h_{x}$ and $h_{y}$ tend to zero. Hence, the widely used direct solvers for the linear system (7) require $O\left(N^{2}\right)$ memory and $O\left(N^{3}\right)$ computational work. Moreover, because of the singularity of the kernel function in the peridynamic model (1), the numerical integration of the nonzero entries of the stiffness matrix $\mathbf{A}_{2 N}$ is involved and expensive. The evaluation and assembly of the $O\left(N^{2}\right)$ nonzero entries of the stiffness matrix $\mathbf{A}_{2 N}$ often constitutes a major portion of the overall computations.

\section{Analysis of $A_{2 N} u_{2 N}$ for any vector $u_{2 N} \in \mathbb{R}^{2 N}$}

A conventional evaluation of the matrix-vector multiplication $\mathbf{A}_{2 N} \mathbf{u}_{2 N}$ in any Krylov subspace iterative method has a computational complexity 
of $O\left(N^{2}\right)$ and a memory requirement of $O\left(N^{2}\right)$, while all other operations have a linear computational complexity. Therefore, to develop a fast Krylov subspace iterative method we need to design an efficient storage mechamism for the stiffness matrix $\mathbf{A}_{2 N}$ and a fast matrix-vector multiplication algorithm to evaluate $\mathbf{A}_{2 N} \mathbf{u}_{2 N}$ for any vector $\mathbf{u}_{2 N} \in \mathbb{R}^{2 N}$.

Due to the coupling between the $x$ component $v$ and the $y$ component $w$ of the displacement $\mathbf{u}_{2 N}$ introduced by the tensor operator $\mathbf{C}\left(\mathbf{x}^{\prime}, \mathbf{x}\right)$ in (2), the stiffness matrix $\mathbf{A}_{2 N}$ in (9) is not BTTB. To develop a fast method, we analyze the displacement vector $\mathbf{u}_{2 N}$ and the stiffness matrix $\mathbf{A}_{2 N}$.

We first decompose the displacement vector $\mathbf{u}_{2 N}$ as the following two auxiliary vectors, which replace the even or odd entries of $\mathbf{u}_{2 N}$ by 0 , respectively

$$
\begin{aligned}
\mathbf{u}_{2 N}^{o}:= & {\left[v_{1,1}, 0, \ldots, v_{N_{x}-1,1}, 0, v_{1,2}, 0, \ldots, v_{N_{x}-1,2}, 0\right.} \\
& \left.\ldots, v_{1, N_{y}-1}, 0, \ldots, v_{N_{x}-1, N_{y}-1}, 0\right]^{T} \\
\mathbf{u}_{2 N}^{e}:= & {\left[0, w_{1,1}, \ldots, 0, w_{N_{x}-1,1}, 0, w_{1,2}, \ldots, 0, w_{N_{x}-1,2},\right.} \\
& \left.\ldots, 0, w_{1, N_{y}-1}, \ldots, 0, w_{N_{x}-1, N_{y}-1}\right]^{T} .
\end{aligned}
$$

We also introduce two $N$-dimensional vectors $\mathbf{v}_{N}$ and $\mathbf{w}_{N}$ as follows

$$
\begin{aligned}
\mathbf{v}_{N} & :=\left[v_{1,1}, \ldots, v_{N_{x}-1,1}, v_{1,2}, \ldots, v_{N_{x}-1,2}, \ldots, v_{1, N_{y}-1}, \ldots, v_{N_{x}-1, N_{y}-1}\right]^{T}, \\
\mathbf{w}_{N} & :=\left[w_{1,1}, \ldots, w_{N_{x}-1,1}, w_{1,2}, \ldots, w_{N_{x}-1,2}, \ldots, w_{1, N_{y}-1}, \ldots, w_{N_{x}-1, N_{y}-1}\right]^{T} .
\end{aligned}
$$

It is clear that

$$
\mathbf{u}_{2 N}=\mathbf{u}_{2 N}^{o}+\mathbf{u}_{2 N}^{e} .
$$

Moreover, eliminating all the zero entries in the $2 N$-dimensional vectors $\mathbf{u}_{2 N}^{o}$ and $\mathbf{u}_{2 N}^{e}$ naturally yields the $N$-dimensional vectors $\mathbf{v}_{N}$ and $\mathbf{w}_{N}$, respectively.

We accordingly introduce the following two $2 N$-by- $2 N$ matrices $\mathbf{A}_{2 N}^{o}$ and $\mathbf{A}_{2 N}^{e}$, which are obtained by replacing the even column entries or odd column entries of $\mathbf{A}_{2 N}$ by zeros, respectively. Namely,

$$
\mathbf{A}_{2 N}^{o}:=\left(\begin{array}{ccccc}
\mathbf{A}_{1,1}^{o} & \mathbf{A}_{1,2}^{o} & \ldots & \mathbf{A}_{1, N-1}^{o} & \mathbf{A}_{1, N}^{o} \\
\mathbf{A}_{2,1}^{o} & \mathbf{A}_{2,2}^{o} & \ldots & \mathbf{A}_{2, N-1}^{o} & \mathbf{A}_{2, N}^{o} \\
\vdots & \vdots & \ddots & \vdots & \vdots \\
\mathbf{A}_{N-1,1}^{o} & \mathbf{A}_{N-1,2}^{o} & \ldots & \mathbf{A}_{N-1, N-1}^{o} & \mathbf{A}_{N-1, N}^{o} \\
\mathbf{A}_{N, 1}^{o} & \mathbf{A}_{N, 2}^{o} & \ldots & \mathbf{A}_{N, N-1}^{o} & \mathbf{A}_{N, N}^{o}
\end{array}\right),
$$


where each entry of $\mathbf{A}_{2 N}^{o}$ is a 2-by-2 matrix of the form

$$
\mathbf{A}_{m, n}^{o}:=\left(\begin{array}{ll}
A_{m, n}^{v, v} & 0 \\
A_{m, n}^{w, v} & 0
\end{array}\right)
$$

and

$$
\mathbf{A}_{2 N}^{e}:=\left(\begin{array}{ccccc}
\mathbf{A}_{1,1}^{e} & \mathbf{A}_{1,2}^{e} & \ldots & \mathbf{A}_{1, N-1}^{e} & \mathbf{A}_{1, N}^{e} \\
\mathbf{A}_{2,1}^{e} & \mathbf{A}_{2,2}^{e} & \ldots & \mathbf{A}_{2, N-1}^{e} & \mathbf{A}_{2, N}^{e} \\
\vdots & \vdots & \ddots & \vdots & \vdots \\
\mathbf{A}_{N-1,1}^{e} & \mathbf{A}_{N-1,2}^{e} & \ldots & \mathbf{A}_{N-1, N-1}^{e} & \mathbf{A}_{N-1, N}^{e} \\
\mathbf{A}_{N, 1}^{e} & \mathbf{A}_{N, 2}^{e} & \ldots & \mathbf{A}_{N, N-1}^{e} & \mathbf{A}_{N, N}^{e}
\end{array}\right),
$$

where each entry of $\mathbf{A}_{2 N}^{e}$ is a 2-by-2 matrix of the form

$$
\mathbf{A}_{m, n}^{e}:=\left(\begin{array}{cc}
0 & A_{m, n}^{v, w} \\
0 & A_{m, n}^{w, w}
\end{array}\right) .
$$

Finally, we introduce the $N$-by- $N$ matrices $\mathbf{A}_{N}^{v, v}, \mathbf{A}_{N}^{v, w}, \mathbf{A}_{N}^{w, v}$, and $\mathbf{A}_{N}^{w, w}$ by replacing each 2-by-2 block $A_{m, n}$ in the matrix $\mathbf{A}_{2 N}$ in (9) by the corresponding entry $A_{m, n}^{v, v}, A_{m, n}^{v, w}, A_{m, n}^{w, v}$, and $A_{m, n}^{w, w}$, respectively. That is,

$$
\begin{aligned}
\mathbf{A}_{N}^{v, v}:= & \left(\begin{array}{ccccc}
A_{1,1}^{v, v} & A_{1,2}^{v, v} & \ldots & A_{1, N-1}^{v, v} & A_{1, N}^{v, v} \\
A_{2,1}^{v, v} & A_{2,2}^{v, v} & \ldots & A_{2, N-1}^{v, v} & A_{2, N}^{v, v} \\
\vdots & \vdots & \ddots & \vdots & \vdots \\
A_{N-1,1}^{v, v} & A_{N-1,2}^{v, v} & \ldots & A_{N-1, N-1}^{v, v} & A_{N-1, N}^{v, v} \\
A_{N, 1}^{v, v} & A_{N, 2}^{v, v} & \ldots & A_{N, N-1}^{v, v} & A_{N, N}^{v, v}
\end{array}\right), \\
\mathbf{A}_{N}^{v, w}:= & \left(\begin{array}{ccccc}
A_{1,1}^{v, w} & A_{1,2}^{v, w} & \ldots & A_{1, N-1}^{v, w} & A_{1, N}^{v, w} \\
A_{2,1}^{v, w} & A_{2,2}^{v, w} & \ldots & A_{2, N-1}^{v, w} & A_{2, N}^{v, w} \\
\vdots & \vdots & \ddots & \vdots & \vdots \\
A_{N-1,1}^{v, w} & A_{N-1,2}^{v, w} & \ldots & A_{N-1, N-1}^{v, w} & A_{N-1, N}^{v, w} \\
A_{N, 1}^{v, w} & A_{N, 2}^{v, w} & \ldots & A_{N, N-1}^{v, w} & A_{N, N}^{v, w}
\end{array}\right), \\
\mathbf{A}_{N}^{w, w}:= & \left(\begin{array}{ccccc}
A_{1,1}^{w, w} & A_{1,2}^{w, w} & \ldots & A_{1, N-1}^{w, w} & A_{1, N}^{w, w} \\
A_{2,1}^{w, w} & A_{2,2}^{w, w} & \ldots & A_{2, N-1}^{w, w} & A_{2, N}^{w, w} \\
\vdots & \vdots & \ddots & \vdots & \vdots \\
A_{N-1,1}^{w, w} & A_{N-1,2}^{w, w} & \ldots & A_{N-1, N-1}^{w, w} & A_{N-1, N}^{w, w} \\
A_{N, 1}^{w, w} & A_{N, 2}^{w, w} & \ldots & A_{N, N-1}^{w, w} & A_{N, N}^{w, w}
\end{array}\right),
\end{aligned}
$$


and $\mathbf{A}_{N}^{w, v}=\mathbf{A}_{N}^{v, w}$.

With all these preparations, we can decompose the matrix-vector multiplication $\mathbf{A}_{2 N} \mathbf{u}_{2 N}$ as follows

$$
\mathbf{A}_{2 N} \mathbf{u}_{2 N}=\mathbf{A}_{2 N} \mathbf{u}_{2 N}^{o}+\mathbf{A}_{2 N} \mathbf{u}_{2 N}^{e}=\mathbf{A}_{2 N}^{o} \mathbf{u}_{2 N}^{o}+\mathbf{A}_{2 N}^{e} \mathbf{u}_{2 N}^{e} .
$$

In addition, if we collect all the odd numbered rows and all the even numbered rows of $\mathbf{A}_{2 N}^{o} \mathbf{u}_{2 N}^{o}$, we obtain the following matrix-vector multiplications

$$
\mathbf{A}_{N}^{v, v} \mathbf{v}_{N}, \quad \mathbf{A}_{N}^{w, v} \mathbf{v}_{N},
$$

respectively. Similarly, if we collect all the odd numbered rows and all the even numbered rows of $\mathbf{A}_{2 N}^{e} \mathbf{u}_{2 N}^{e}$, we obtain the following matrix-vector multiplications

$$
\mathbf{A}_{N}^{v, w} \mathbf{w}_{N}, \quad \mathbf{A}_{N}^{w, w} \mathbf{w}_{N},
$$

respectively.

In summary, to evaluate the matrix-vector multiplication $\mathbf{A}_{2 N} \mathbf{u}_{2 N}$ efficiently, we need only to efficiently evaluate the matrix-vector multiplications $\mathbf{A}_{N}^{v, v} \mathbf{v}_{N}, \mathbf{A}_{N}^{w, v} \mathbf{v}_{N}, \mathbf{A}_{N}^{v, w} \mathbf{w}_{N}$, and $\mathbf{A}_{N}^{w, w} \mathbf{w}_{N}$. Similarly, to efficiently store and assemble the stiffness matrix $\mathbf{A}_{2 N}$, we need only to efficiently store and assemble the submatrices $\mathbf{A}_{N}^{v, v}, \mathbf{A}_{N}^{w, v}, \mathbf{A}_{N}^{v, w}$, and $\mathbf{A}_{N}^{w, w}$. We will address their efficient evaluation and storage in section 5 .

\section{Structure of matrices $\mathrm{A}_{2 N}, \mathrm{~A}_{N}^{v, v}, \mathrm{~A}_{N}^{v, w}$, and $\mathrm{A}_{N}^{w, w}$}

In this section, we analyze the structure of the matrices $\mathbf{A}_{2 N}, \mathbf{A}_{N}^{v, v}, \mathbf{A}_{N}^{v, w}$ and $\mathbf{A}_{N}^{w, w}$. We begin with the analysis of the structure of the stiffness matrix $\mathbf{A}_{2 N}$.

Theorem 1. Let $K$ and $L$ be defined by (3). The stiffness matrix $\mathbf{A}_{2 N}$ can be expressed as the following block-banded matrix of order $\left(N_{y}-1\right)$ with 
a block bandwidth $2 L+1$

$$
\mathbf{A}_{2 N}=\left(\begin{array}{ccccccccc}
\mathbf{B}^{1,1} & \cdots & \mathbf{B}^{1, L+1} & \mathbf{0} & \cdots & \mathbf{0} & \mathbf{0} & \cdots & \mathbf{0} \\
\vdots & \ddots & \ddots & \ddots & \ddots & \ddots & \ddots & \ddots & \vdots \\
\mathbf{B}^{L+1,1} & \ddots & \mathbf{B}^{L+1, L+1} & \ddots & \ddots & \mathbf{0} & \ddots & \ddots & \mathbf{0} \\
\mathbf{0} & \ddots & \ddots & \mathbf{B}^{L+2, L+2} & \ddots & \ddots & \mathbf{0} & \ddots & \mathbf{0} \\
\vdots & \ddots & \ddots & \ddots & \ddots & \ddots & \ddots & \ddots & \vdots \\
\mathbf{0} & \ddots & \mathbf{0} & \ddots & \ddots & \ddots & \ddots & \ddots & \mathbf{0} \\
\mathbf{0} & \ddots & \ddots & \mathbf{0} & \ddots & \ddots & \mathbf{B}^{N_{y}-L-1, N_{y}-L-1} & \ddots & \mathbf{B}^{N_{y}-L-1, N_{y}-1} \\
\vdots & \ddots & \ddots & \ddots & \ddots & \ddots & \ddots & \ddots & \vdots \\
\mathbf{0} & \cdots & \mathbf{0} & \mathbf{0} & \cdots & \mathbf{0} & \mathbf{B}^{N_{y}-1, N_{y}-L-1} & \cdots & \mathbf{B}^{N_{y}-i, N_{y}-1}
\end{array}\right) .
$$

Furthermore, each matrix block $\mathbf{B}^{j, j^{\prime}}$ is of order $2\left(N_{x}-1\right)$. For $\left|j-j^{\prime}\right| \leqslant L$, all the matrix blocks $\mathbf{B}^{j j^{\prime}}$ can be expressed as

$$
\mathbf{B}^{j, j^{\prime}}=\left(\begin{array}{ccccccccc}
\mathbf{B}_{1,1}^{j, j^{\prime}} & \cdots & \mathbf{B}_{1, K+1}^{j, j^{\prime}} & \mathbf{0} & \cdots & \mathbf{0} & \mathbf{0} & \cdots & \mathbf{0} \\
\vdots & \ddots & \ddots & \ddots & \ddots & \ddots & \ddots & \ddots & \vdots \\
\mathbf{B}_{K+1,1}^{j, j^{\prime}} & \ddots & \mathbf{B}_{K+1, K+1}^{j, j^{\prime}} & \ddots & \ddots & \mathbf{0} & \ddots & \ddots & \mathbf{0} \\
\mathbf{0} & \ddots & \ddots & \mathbf{B}_{K+2, K+2}^{j, j^{\prime}} & \ddots & \ddots & \mathbf{0} & \ddots & \mathbf{0} \\
\vdots & \ddots & \ddots & \ddots & \ddots & \ddots & \ddots & \ddots & \vdots \\
\mathbf{0} & \ddots & \mathbf{0} & \ddots & \ddots & \ddots & \ddots & \ddots & \mathbf{0} \\
\mathbf{0} & \ddots & \ddots & \mathbf{0} & \ddots & \ddots & \mathbf{B}_{N_{x}-K-1, N_{x}-K-1}^{j, j^{\prime}} & \ddots & \mathbf{B}_{N_{x}-K-1, N_{x}-1}^{j, j^{\prime}} \\
\vdots & \ddots & \ddots & \ddots & \ddots & \ddots & \ddots & \ddots & \vdots \\
\mathbf{0} & \cdots & \mathbf{0} & \mathbf{0} & \cdots & \mathbf{0} & \mathbf{B}_{N_{x}-1, N_{x}-K-1}^{j, j^{\prime}} & \cdots & \mathbf{B}_{N_{x}-1, N_{x}-1}^{j, j^{\prime}}
\end{array}\right) .
$$

Finally, for $\left|j^{\prime}-j\right| \leqslant L$ and $\left|i^{\prime}-i\right| \leqslant K$, each 2-by-2 matrix block satisfies

$$
\mathbf{B}_{i, i^{\prime}}^{j, j^{\prime}}=\mathbf{A}_{m, n},
$$

where the indices $i, i^{\prime}, j$, and $j^{\prime}$ and the indices $m$ and $n$ are related by (12).

Proof. We first rewrite the stiffness matrix as the following form

$$
\mathbf{A}_{2 N}=\left(\begin{array}{cccc}
\mathbf{B}^{1,1} & \mathbf{B}^{1,2} & \cdots & \mathbf{B}^{1, N_{y}-1} \\
\mathbf{B}^{2,1} & \mathbf{B}^{2,2} & \ddots & \mathbf{B}^{2, N_{y}-1} \\
\vdots & \ddots & \ddots & \vdots \\
\mathbf{B}^{N_{y}-1,1} & \mathbf{B}^{N_{y}-1,2} & \cdots & \mathbf{B}^{N_{y}-1, N_{y}-1}
\end{array}\right)
$$


Here each matrix block $\mathbf{B}^{j, j^{\prime}}$ of order $2\left(N_{x}-1\right)$ represents the interaction of row $j$ and row $j^{\prime}$ in the discrete system for $1 \leqslant j \leqslant N_{y}-1$ and $1 \leqslant j^{\prime} \leqslant N_{y}-1$. Furthermore, each matrix block $\mathbf{B}^{j, j^{\prime}}$ can be expressed as

$$
\mathbf{B}^{j, j^{\prime}}=\left(\begin{array}{cccc}
\mathbf{B}_{1,1}^{j, j^{\prime}} & \mathbf{B}_{1,2}^{j, j^{\prime}} & \cdots & \mathbf{B}_{1, N_{x}-1}^{j, j^{\prime}} \\
\mathbf{B}_{2,1}^{j, j^{\prime}} & \mathbf{B}_{2,2}^{j, j^{\prime}} & \ddots & \mathbf{B}_{2, N_{x}-1}^{j, j^{\prime}} \\
\vdots & \ddots & \ddots & \vdots \\
\mathbf{B}_{N_{x}-1,1}^{j, j^{\prime}} & \mathbf{B}_{N_{x}-1,2}^{j, j^{\prime}} & \cdots & \mathbf{B}_{N_{x}-1, N_{x}-1}^{j, j^{\prime}}
\end{array}\right) .
$$

Here each matrix block $\mathbf{B}_{i, i^{\prime}}^{j, j^{\prime}}$ of order 2 represents the interaction between the $i$ th node in the $\mathrm{j}$ row of the grid and the $i^{\prime}$ th node in the $j^{\prime}$ row of the grid for $1 \leqslant i \leqslant N_{x}-1$ and $1 \leqslant i^{\prime} \leqslant N_{x}-1$. In addition, we have

$$
\mathbf{B}_{i, i^{\prime}}^{j, j^{\prime}}=\mathbf{A}_{m, n},
$$

where the indices $i, i^{\prime}, j$, and $j^{\prime}$ and the indices $m$ and $n$ are related by (12). Thus to prove Theorem 1, it remains to prove the matrix blocks $\mathbf{B}^{j, j^{\prime}}$ with $\left|j-j^{\prime}\right| \geqslant L+1$ are zeros and the matrix blocks $\mathbf{B}_{i, i^{\prime}}^{j, j^{\prime}}$ with $\left|j-j^{\prime}\right| \leqslant L$ and $\left|i-i^{\prime}\right| \geqslant K+1$ are zeros.

We conclude from the expressions of the entries of the matrix $\mathbf{A}_{2 N}$ in (11) and the relations between indices in (12) that the matrix blocks $\mathbf{A}_{m, n}=0$ if

$$
\operatorname{supp}\left(\phi_{i^{\prime}, j^{\prime}}\right) \cap B_{\delta}\left(x_{i}, y_{j}\right)=\emptyset .
$$

We deduce from the definition $L$ in (3) that (33) holds for $\left|j^{\prime}-j\right| \geqslant L+1$. Thus, the matrix (30) can be expressed in the form of (27).

We next investigate the structure of each block matrix $\mathbf{B}^{i, j}$ with $\left|j^{\prime}-j\right| \leqslant$ $L$. We again use the definition $K$ in (3) to conclude that (33) holds for $\left|i^{\prime}-i\right| \geqslant K+1$ and thus the matrix (31) can be expressed in the form of (28).

The equation (29) can be directly obtained from (32) for $\left|i-i^{\prime}\right| \leqslant K$ and $\left|j-j^{\prime}\right| \leqslant L$.

Theorem 2. Each of the matrices $\mathbf{A}_{N}^{v, v}, \mathbf{A}_{N}^{v, w}$ and $\mathbf{A}_{N}^{w, w}$ can be expressed as an $\left(N_{y}-1\right)$-by-( $\left.N_{y}-1\right)$ block-banded Toeplitz matrix with a block bandwidth 
$2 L+1$

$$
\mathbf{A}_{N}^{I}=\left(\begin{array}{ccccccccc}
\mathbf{T}_{0}^{I} & \cdots & \mathbf{T}_{L}^{I} & \mathbf{0} & \cdots & \mathbf{0} & \mathbf{0} & \cdots & \mathbf{0} \\
\vdots & \ddots & \ddots & \ddots & \ddots & \ddots & \ddots & \ddots & \vdots \\
\mathbf{T}_{-L}^{I} & \ddots & \mathbf{T}_{0}^{I} & \ddots & \ddots & \mathbf{0} & \ddots & \ddots & \mathbf{0} \\
\mathbf{0} & \ddots & \ddots & \mathbf{T}_{0}^{I} & \ddots & \ddots & \mathbf{0} & \ddots & \mathbf{0} \\
\vdots & \ddots & \ddots & \ddots & \ddots & \ddots & \ddots & \ddots & \vdots \\
\mathbf{0} & \ddots & \mathbf{0} & \ddots & \ddots & \mathbf{T}_{0}^{I} & \ddots & \ddots & \mathbf{0} \\
\mathbf{0} & \ddots & \ddots & \mathbf{0} & \ddots & \ddots & \mathbf{T}_{0}^{I} & \ddots & \mathbf{T}_{L}^{I} \\
\vdots & \ddots & \ddots & \ddots & \ddots & \ddots & \ddots & \ddots & \vdots \\
\mathbf{0} & \cdots & \mathbf{0} & \mathbf{0} & \cdots & \mathbf{0} & \mathbf{T}_{-L}^{I} & \cdots & \mathbf{T}_{0}^{I}
\end{array}\right)
$$

where the superscript $I=(v, v),(v, w)$ or $(w, w)$. Furthermore, each matrix block $\mathbf{T}_{j}^{I}$, with $-L \leqslant j \leqslant L$, is an $\left(N_{x}-1\right)$-by- $\left(N_{x}-1\right)$ banded Toeplitz matrix with a bandwidth $2 K+1$

$$
\mathbf{T}_{j}^{I}=\left(\begin{array}{ccccccccc}
t_{0, j}^{I} & \cdots & t_{K, j}^{I} & 0 & \cdots & 0 & 0 & \cdots & 0 \\
\vdots & \ddots & \ddots & \ddots & \ddots & \ddots & \ddots & \ddots & \vdots \\
t_{-K, j}^{I} & \ddots & t_{0, j}^{I} & \ddots & \ddots & 0 & \ddots & \ddots & 0 \\
0 & \ddots & \ddots & t_{0, j}^{I} & \ddots & \ddots & 0 & \ddots & 0 \\
\vdots & \ddots & \ddots & \ddots & \ddots & \ddots & \ddots & \ddots & \vdots \\
0 & \ddots & 0 & \ddots & \ddots & t_{0, j}^{I} & \ddots & \ddots & 0 \\
0 & \ddots & \ddots & 0 & \ddots & \ddots & t_{0, j}^{I} & \ddots & t_{K, j}^{I} \\
\vdots & \ddots & \ddots & \ddots & \ddots & \ddots & \ddots & \ddots & \vdots \\
0 & \cdots & 0 & 0 & \cdots & 0 & t_{-K, j}^{I} & \cdots & t_{0, j}^{I}
\end{array}\right) .
$$

Proof. We need only to prove (34)-(35) for $\mathbf{A}_{N}^{v, v}$. The proofs for $\mathbf{A}_{N}^{v, w}$ and $\mathbf{A}_{N}^{w, w}$ are similar. By Theorem $1, \mathbf{A}_{2 N}$ is a $(2 L+1)$ block-banded, $(2 K+1)$ block-banded block matrix in which each matrix block is a matrix of order 2. Following the proof of Theorem 1 and the definition (21), the matrix $\mathbf{A}_{N}^{v, v}$ is a $(2 L+1)$ block-banded, $(2 K+1)$ banded matrix. More precisely, $\mathbf{A}_{N}^{v, v}$ is 
of the form

$$
\mathbf{A}_{N}^{v, v}=\left(\begin{array}{ccccccccc}
\mathbf{B}_{v}^{1,1} & \cdots & \mathbf{B}_{v}^{1, L+1} & \mathbf{0} & \cdots & \mathbf{0} & \mathbf{0} & \cdots & \mathbf{0} \\
\vdots & \ddots & \ddots & \ddots & \ddots & \ddots & \ddots & \ddots & \vdots \\
\mathbf{B}_{v}^{L+1,1} & \ddots & \mathbf{B}_{v}^{L+1, L+1} & \ddots & \ddots & \mathbf{0} & \ddots & \ddots & \mathbf{0} \\
\mathbf{0} & \ddots & \ddots & \mathbf{B}_{v}^{L+2, L+2} & \ddots & \ddots & \mathbf{0} & \ddots & \mathbf{0} \\
\vdots & \ddots & \ddots & \ddots & \ddots & \ddots & \ddots & \ddots & \vdots \\
\mathbf{0} & \ddots & \mathbf{0} & \ddots & \ddots & \ddots & \ddots & \ddots & \mathbf{0} \\
\mathbf{0} & \ddots & \ddots & \mathbf{0} & \ddots & \ddots & \mathbf{B}_{v}^{N_{y}-L-1, N_{y}-L-1} & \ddots & \mathbf{B}_{v}^{N_{y}-L-1, N_{y}-1} \\
\vdots & \ddots & \ddots & \ddots & \ddots & \ddots & \ddots & \ddots & \vdots \\
\mathbf{0} & \cdots & \mathbf{0} & \mathbf{0} & \cdots & \mathbf{0} & \mathbf{B}_{v}^{N_{y}-1, N_{y}-L-1} & \cdots & \mathbf{B}_{v}^{N_{y}-1, N_{y}-1}
\end{array}\right),
$$

where each matrix block $\mathbf{B}_{v}^{j, j^{\prime}}$ is a banded matrix with a bandwidth $2 K+1$

$$
\mathbf{B}_{v}^{j, j^{\prime}}=\left(\begin{array}{ccccccccc}
b_{1,1}^{j, j^{\prime}} & \cdots & b_{1, K+1}^{j, j^{\prime}} & 0 & \cdots & 0 & 0 & \cdots & 0 \\
\vdots & \ddots & \ddots & \ddots & \ddots & \ddots & \ddots & \ddots & \vdots \\
b_{K+1,1}^{j, j^{\prime}} & \ddots & b_{K+1, K+1}^{j, j^{\prime}} & \ddots & \ddots & 0 & \ddots & \ddots & 0 \\
0 & \ddots & \ddots & b_{K+2, K+2}^{j, j^{\prime}} & \ddots & \ddots & 0 & \ddots & 0 \\
\vdots & \ddots & \ddots & \ddots & \ddots & \ddots & \ddots & \ddots & \vdots \\
0 & \ddots & 0 & \ddots & \ddots & \ddots & \ddots & \ddots & 0 \\
0 & \ddots & \ddots & 0 & \ddots & \ddots & b_{N_{x}-K-1, N_{x}-K-1}^{j, j^{\prime}} & \ddots & b_{N_{x}-K-1, N_{x}-1}^{j, j^{\prime}} \\
\vdots & \ddots & \ddots & \ddots & \ddots & \ddots & \ddots & \ddots & \vdots \\
0 & \cdots & 0 & 0 & \cdots & 0 & b_{N_{x}-1, N_{x}-K-1}^{j, j^{\prime}} & \cdots & b_{N_{x}-1, N_{x}-1}^{j, j^{\prime}}
\end{array}\right)
$$

and

$$
b_{i, i^{\prime}}^{j, j^{\prime}}=A_{m, n}^{v, v}, \quad\left|i-i^{\prime}\right| \leqslant K, \quad\left|j-j^{\prime}\right| \leqslant L,
$$

where the indices $i, i^{\prime}, j$, and $j^{\prime}$, and the indices $m$ and $n$ are related by (12).

By the following translations

$$
\xi_{1}=x^{\prime}-x_{i}, \quad \xi_{2}=y^{\prime}-y_{j},
$$

the pyramid function $\phi_{i^{\prime}, j^{\prime}}\left(x^{\prime}, y^{\prime}\right)$ defined in (4) can be expressed as

$$
\begin{aligned}
\phi_{i^{\prime}, j^{\prime}}\left(x^{\prime}, y^{\prime}\right) & =\psi\left(\frac{x^{\prime}-x_{i^{\prime}}}{h_{x}}\right) \psi\left(\frac{y^{\prime}-y_{j^{\prime}}}{h_{y}}\right) \\
& =\psi\left(\frac{\xi_{1}-x_{i^{\prime}-i}}{h_{x}}\right) \psi\left(\frac{\xi_{2}-y_{j^{\prime}-j}}{h_{y}}\right)=\phi_{i^{\prime}-i, j^{\prime}-j}\left(\xi_{1}, \xi_{2}\right) .
\end{aligned}
$$


Therefore, the first equation in (11) can be deduced to

$$
A_{m, n}^{v, v}=\int_{B_{\delta}(0,0)} \frac{\xi_{1}{ }^{2}}{\xi_{1}^{2}+\xi_{2}^{2}} \sigma\left(\left\|\left(\xi_{1}, \xi_{2}\right)\right\|\right)\left(\delta_{m, n}-\phi_{i^{\prime}-i, j^{\prime}-j}\left(\xi_{1}, \xi_{2}\right)\right) d \xi_{1} d \xi_{2} .
$$

To prove the block-Toeplitz property of the matrix $\mathbf{A}_{N}^{v, v}$, we consider the matrix blocks $\mathbf{B}_{v}^{j_{1}, j_{1}^{\prime}}$ and $\mathbf{B}_{v}^{j_{2}, j_{2}^{\prime}}$ in (36) that are on the same diagonal. Namely, their superscripts $j_{1}$ and $j_{1}^{\prime}$ as well as $j_{2}$ and $j_{2}^{\prime}$ satisfy

$$
j_{1}^{\prime}-j_{1}=j_{2}^{\prime}-j_{2}=l
$$

for some $-L \leqslant l \leqslant L$. Let

$$
\begin{array}{ll}
m_{1}:=\left(j_{1}-1\right)\left(N_{x}-1\right)+i, & 1 \leqslant i \leqslant N_{x}-1,1 \leqslant j_{1} \leqslant N_{y}-1, \\
n_{1}:=\left(j_{1}^{\prime}-1\right)\left(N_{x}-1\right)+i^{\prime}, & 1 \leqslant i^{\prime} \leqslant N_{x}-1,1 \leqslant j_{1}^{\prime} \leqslant N_{y}-1, \\
m_{2}:=\left(j_{2}-1\right)\left(N_{x}-1\right)+i, & 1 \leqslant i \leqslant N_{x}-1,1 \leqslant j_{2} \leqslant N_{y}-1, \\
n_{2}:=\left(j_{2}^{\prime}-1\right)\left(N_{x}-1\right)+i^{\prime}, & 1 \leqslant i^{\prime} \leqslant N_{x}-1,1 \leqslant j_{2}^{\prime} \leqslant N_{y}-1 .
\end{array}
$$

Then, we use (38) and (41) to prove that for $1 \leqslant i, i^{\prime} \leqslant N_{x}-1$

$$
\begin{aligned}
b_{i, i^{\prime}}^{j_{1}, j_{1}^{\prime}} & =A_{m_{1}, n_{1}}^{v, v} \\
& =\int_{B_{\delta}(0,0)} \frac{\xi_{1}{ }^{2}}{\xi_{1}{ }^{2}+\xi_{2}{ }^{2}} \sigma\left(\left\|\left(\xi_{1}, \xi_{2}\right)\right\|\right)\left(\delta_{m_{1}, n_{1}}-\phi_{i^{\prime}-i, j_{1}^{\prime}-j_{1}}\left(\xi_{1}, \xi_{2}\right)\right) d \xi_{1} d \xi_{2} \\
& =\int_{B_{\delta}(0,0)} \frac{\xi_{1}{ }^{2}}{\xi_{1}{ }^{2}+\xi_{2}{ }^{2}} \sigma\left(\left\|\left(\xi_{1}, \xi_{2}\right)\right\|\right)\left(\delta_{m_{2}, n_{2}}-\phi_{i^{\prime}-i, j_{2}^{\prime}-j_{2}}\left(\xi_{1}, \xi_{2}\right)\right) d \xi_{1} d \xi_{2} \\
& =A_{m_{2}, n_{2}}^{v, v}=b_{i, i^{\prime}}^{j_{2}, j_{2}^{\prime}} .
\end{aligned}
$$

Therefore, we have proved that

$$
\mathbf{B}_{v}^{j_{1}, j_{1}^{\prime}}=\mathbf{B}_{v}^{j_{2}, j_{2}^{\prime}},
$$

if the matrix blocks $\mathbf{B}_{v}^{j_{1}, j_{1}^{\prime}}$ and $\mathbf{B}_{v}^{j_{2}, j_{2}^{\prime}}$ are on the same diagonal in (36). Accordingly, we have proved that $\mathbf{A}_{N}^{v, v}$ is a block-banded Toeplitz matrix with a block bandwidth $2 L+1$ and the matrix (36) can be expressed in the form of (34).

Next, we fix each block matrix $\mathbf{B}_{v}^{j, j^{\prime}}$ with $\left|j^{\prime}-j\right| \leqslant L$ and look at their entries $b_{i_{3}, i_{3}^{\prime}}^{j, j^{\prime}}$ and $b_{i_{4}, i_{4}^{\prime}}^{j, j^{\prime}}$ that are on the same diagonal. Namely, their subscripts $i_{3}$ and $i_{3}^{\prime}$ as well as $i_{4}$ and $i_{4}^{\prime}$ satisfy

$$
i_{3}^{\prime}-i_{3}=i_{4}^{\prime}-i_{4}=k
$$


for some $-K \leqslant k \leqslant K$. Let

$$
\begin{array}{ll}
m_{3}:=(j-1)\left(N_{x}-1\right)+i_{3}, & 1 \leqslant i_{3} \leqslant N_{x}-1,1 \leqslant j \leqslant N_{y}-1, \\
n_{3}:=\left(j^{\prime}-1\right)\left(N_{x}-1\right)+i_{3}^{\prime}, & 1 \leqslant i_{3}^{\prime} \leqslant N_{x}-1,1 \leqslant j^{\prime} \leqslant N_{y}-1, \\
m_{4}:=(j-1)\left(N_{x}-1\right)+i_{4}, & 1 \leqslant i_{4} \leqslant N_{x}-1,1 \leqslant j \leqslant N_{y}-1, \\
n_{4}:=\left(j^{\prime}-1\right)\left(N_{x}-1\right)+i_{4}^{\prime}, & 1 \leqslant i_{4}^{\prime} \leqslant N_{x}-1,1 \leqslant j^{\prime} \leqslant N_{y}-1 .
\end{array}
$$

We again apply (38) to obtain

$$
\begin{aligned}
b_{i_{3}, i_{3}^{\prime}}^{j, j^{\prime}} & =A_{m_{3}, n_{3}}^{v, v} \\
& =\int_{B_{\delta}(0,0)} \frac{\xi_{1}^{2}}{\xi_{1}^{2}+\xi_{2}^{2}} \sigma\left(\left\|\left(\xi_{1}, \xi_{2}\right)\right\|\right)\left(\delta_{m_{3}, n_{3}}-\phi_{i_{3}^{\prime}-i_{3}, j^{\prime}-j}\left(\xi_{1}, \xi_{2}\right)\right) d \xi_{1} d \xi_{2} \\
& =\int_{B_{\delta}(0,0)} \frac{\xi_{1}{ }^{2}}{\xi_{1}{ }^{2}+\xi_{2}{ }^{2}} \sigma\left(\left\|\left(\xi_{1}, \xi_{2}\right)\right\|\right)\left(\delta_{m_{4}, n_{4}}-\phi_{i_{4}^{\prime}-i_{4}, j^{\prime}-j}\left(\xi_{1}, \xi_{2}\right)\right) d \xi_{1} d \xi_{2} \\
& =A_{m_{4}, n_{4}}^{v, v}=b_{i_{4}, i_{4}^{\prime}}^{j, j^{\prime}}
\end{aligned}
$$

Hence we conclude that each matrix block $\mathbf{B}_{v}^{j, j^{\prime}}$ in (36) with $\left|j-j^{\prime}\right| \leqslant L$ is a $(2 K+1)$ banded Toeplitz matrix and that the matrix (37) can be expressed in the form of (35).

\section{Efficient storage and assembly of the stiffness matrix and a fast Krylov subspace iterative method}

We utilize the structure of the matrices $\mathbf{A}_{N}^{v, v}, \mathbf{A}_{N}^{v, w}$, and $\mathbf{A}_{N}^{w, w}$ proved in section 4 to develop an efficient storage and assembly mechanism for the stiffness matrix $\mathbf{A}_{2 N}$.

Theorem 3. The evaluation and storage of each of the matrices $\mathbf{A}_{N}^{v, v}$, $\mathbf{A}_{N}^{v, w}$, and $\mathbf{A}_{N}^{w, w}$ can be accomplished by evaluating and storing only $(2 K+$ $1)(2 L+1)$ entries. More precisely, we need only to evaluate and store the following matrices of order $(2 K+1)(2 L+1)$

$$
G^{v, v}:=\left(\begin{array}{ccccc}
t_{-K,-L}^{v, v} & \cdots & t_{-K, 0}^{v, v} & \cdots & t_{-K, L}^{v, v} \\
\vdots & \ddots & \vdots & \ddots & \vdots \\
t_{0,-L}^{v, v} & \cdots & t_{0,0}^{v, v} & \cdots & t_{0, L}^{v, v} \\
\vdots & \ddots & \vdots & \ddots & \vdots \\
t_{K,-L}^{v, v} & \cdots & t_{K, 0}^{v, v} & \cdots & t_{K, L}^{v, v}
\end{array}\right),
$$


and

$$
G^{v, w}:=\left(\begin{array}{ccccc}
t_{-K,-L}^{v, w} & \cdots & t_{-K, 0}^{v, w} & \cdots & t_{-K, L}^{v, w} \\
\vdots & \ddots & \vdots & \ddots & \vdots \\
t_{0,-L}^{v, w} & \cdots & t_{0,0}^{v, w} & \cdots & t_{0, L}^{v, w} \\
\vdots & \ddots & \vdots & \ddots & \vdots \\
t_{K,-L}^{v, w} & \cdots & t_{K, 0}^{v, w} & \cdots & t_{K, L}^{v, w}
\end{array}\right)
$$

$$
G^{w, w}:=\left(\begin{array}{ccccc}
t_{-K,-L}^{w, w} & \cdots & t_{-K, 0}^{w, w} & \cdots & t_{-K, L}^{w, w} \\
\vdots & \ddots & \vdots & \ddots & \vdots \\
t_{0,-L}^{w, w} & \cdots & t_{0,0}^{w, w} & \cdots & t_{0, L}^{w, w} \\
\vdots & \ddots & \vdots & \ddots & \vdots \\
t_{K,-L}^{w, w} & \cdots & t_{K, 0}^{w, w} & \cdots & t_{K, L}^{w, w}
\end{array}\right) .
$$

Proof. We need only to prove the theorem for the matrix $\mathbf{A}_{N}^{v, v}$. The proofs for $\mathbf{A}_{N}^{v, w}$ and $\mathbf{A}_{N}^{w, w}$ are similar. By the expression (34) in Theorem 2, to evaluate and store the matrix $\mathbf{A}_{N}^{v, v}$ we need only to evaluate and store the $2 L+1$ matrices $\mathbf{T}_{l}^{v, v}$ for $-L \leqslant l \leqslant L$. By the expression (35) in Theorem 2 , to evaluate and store each matrix $\mathbf{T}_{l}^{v, v}$, we need only to evaluate and store the entries $t_{k, l}^{v, v}$ for $-K \leqslant k \leqslant K$ and $-L \leqslant l \leqslant L$.

Remark 1. The indices $(k, l)$ represents the relative positions of points $\left(x_{i}, y_{j}\right)$ and $\left(x_{i^{\prime}}, y_{j^{\prime}}\right)$ for $\left|i-i^{\prime}\right| \leqslant K$ and $\left|j-j^{\prime}\right| \leqslant L$, i.e., $k=i^{\prime}-i$ and $l=j^{\prime}-j$. Therefore, we have $t_{k, l}^{I}=t_{i^{\prime}-i, j^{\prime}-j}^{I}=b_{i, i^{\prime}}^{j, j^{\prime}}=A_{m, n}^{I}$ with $I=(v, v)$, $(v, w)$, and $(w, w)$, where the second equality is obtained from the fact that (35) is equal to (37), the third equality is obtained by (38) or (29) and the indices $i, i^{\prime}, j$, and $j^{\prime}$ as well as the indices $m$ and $n$ are related by (12).

Remark 2. The mechanism in Theorem 3 reduces the evaluation and storage of the stiffness matrices $\mathbf{A}_{N}^{v, v}, \mathbf{A}_{N}^{v, w}$ and $\mathbf{A}_{N}^{w, w}$ from $O\left(N^{2}\right)$ to $O((2 K+$ 1) $(2 L+1))=O\left(\delta^{2} /\left(h_{x} h_{y}\right)\right)=O\left(N_{x} N_{y}\right)=O(N)$ for a fixed $\delta$. Hence, the stiffness matrix $\mathbf{A}_{2 N}$ can be stored in $O(N)$ memory instead of $O\left(N^{2}\right)$ memory and can be assembled by evaluating only $O(N)$ entries instead of $O\left(N^{2}\right)$ entries.

Theorem 4. For any vector $\mathbf{d}_{N} \in \mathbb{R}^{N}$, the matrix-vector multiplications $\mathbf{A}_{N}^{v, v} \mathbf{d}_{N}, \mathbf{A}_{N}^{v, w} \mathbf{d}_{N}$, and $\mathbf{A}_{N}^{w, w} \mathbf{d}_{N}$ can be evaluated in $O(N \log N)$ operations.

Proof. We need only to prove that $\mathbf{A}_{N}^{v, v} \mathbf{d}_{N}$ can be evaluated in $O(N \log N)$ operations. $\mathbf{A}_{N}^{v, w} \mathbf{d}_{N}$ and $\mathbf{A}_{N}^{v, v} \mathbf{d}_{N}$ can be computed by the similar way. By Theorem 2, $\mathbf{A}_{N}^{v, v}$ is BTTB. Then each Toeplitz matrix $\mathbf{T}_{j}^{v, v}$ of order $N_{x}-1$ in 
(34) can be embedded into a $2\left(N_{x}-1\right)$-by-2( $\left.N_{x}-1\right)$ circulant matrix $[6,15]$

$$
\mathbf{C}_{j}^{v, v}:=\left(\begin{array}{cc}
\mathbf{T}_{j}^{v, v} & \tilde{\mathbf{T}}_{j}^{v, v} \\
\tilde{\mathbf{T}}_{j}^{v, v} & \mathbf{T}_{j}^{v, v}
\end{array}\right),
$$

where the matrix $\tilde{\mathbf{T}}_{j}^{v, v}$ is defined by

$$
\tilde{\mathbf{T}}_{j}^{v, v}:=\left(\begin{array}{ccccccccc}
0 & \cdots & 0 & 0 & \cdots & 0 & t_{-K, j}^{v, v} & \cdots & t_{-1, j}^{v, v} \\
\vdots & \ddots & \ddots & \ddots & \ddots & \ddots & \ddots & \ddots & \vdots \\
0 & \ddots & 0 & 0 & \ddots & 0 & \ddots & \ddots & t_{-K, j}^{v, v} \\
0 & \ddots & 0 & 0 & \ddots & \ddots & 0 & \ddots & 0 \\
\vdots & \ddots & \ddots & \ddots & \ddots & \ddots & \ddots & \ddots & \vdots \\
0 & \ddots & 0 & \ddots & \ddots & \ddots & \ddots & \ddots & 0 \\
t_{K, j}^{v, v} & \ddots & \ddots & 0 & \ddots & \ddots & 0 & \ddots & 0 \\
\vdots & \ddots & \ddots & \ddots & \ddots & \ddots & \ddots & \ddots & \vdots \\
t_{1, j}^{v, v} & \cdots & t_{K, j}^{v, v} & 0 & \cdots & 0 & 0 & \cdots & 0
\end{array}\right) .
$$

Replacing each $\mathbf{T}_{j}^{v, v}$ in (34) by the corresponding circulant matrix $\mathbf{C}_{j}^{v, v}$, we obtain a block-Toeplitz-circulant-block (BTCB) matrix $\mathbf{D}^{v, v}$

$$
\mathbf{D}^{v, v}:=\left(\begin{array}{ccccccccc}
\mathbf{C}_{0}^{v, v} & \cdots & \mathbf{C}_{L}^{v, v} & \mathbf{0} & \cdots & \mathbf{0} & \mathbf{0} & \cdots & \mathbf{0} \\
\vdots & \ddots & \ddots & \ddots & \ddots & \ddots & \ddots & \ddots & \vdots \\
\mathbf{C}_{-L}^{v, v} & \ddots & \mathbf{C}_{0}^{v, v} & \ddots & \ddots & \mathbf{0} & \ddots & \ddots & \mathbf{0} \\
\mathbf{0} & \ddots & \ddots & \mathbf{C}_{0}^{v, v} & \ddots & \ddots & \mathbf{0} & \ddots & \mathbf{0} \\
\vdots & \ddots & \ddots & \ddots & \ddots & \ddots & \ddots & \ddots & \vdots \\
\mathbf{0} & \ddots & \mathbf{0} & \ddots & \ddots & \mathbf{C}_{0}^{v, v} & \ddots & \ddots & \mathbf{0} \\
\mathbf{0} & \ddots & \ddots & \mathbf{0} & \ddots & \ddots & \mathbf{C}_{0}^{v, v} & \ddots & \mathbf{C}_{L}^{v, v} \\
\vdots & \ddots & \ddots & \ddots & \ddots & \ddots & \ddots & \ddots & \vdots \\
\mathbf{0} & \cdots & \mathbf{0} & \mathbf{0} & \cdots & \mathbf{0} & \mathbf{C}_{-L}^{v, v} & \cdots & \mathbf{C}_{0}^{v, v}
\end{array}\right) .
$$

Then $\mathbf{D}^{v, v}$ can be embedded into a $2\left(N_{y}-1\right)$-by-2( $\left.N_{y}-1\right)$ circulant block matrix $\mathbf{C}^{v, v}$ as follows $[6,15]$

$$
\mathbf{C}^{v, v}:=\left(\begin{array}{cc}
\mathbf{D}^{v, v} & \tilde{\mathbf{D}}^{v, v} \\
\tilde{\mathbf{D}}^{v, v} & \mathbf{D}^{v, v}
\end{array}\right)
$$


where

$$
\tilde{\mathbf{D}}^{v, v}:=\left(\begin{array}{ccccccccc}
\mathbf{0} & \cdots & \mathbf{0} & \mathbf{0} & \cdots & \mathbf{0} & \mathbf{C}_{-L}^{v, v} & \cdots & \mathbf{C}_{-1}^{v, v} \\
\vdots & \ddots & \ddots & \ddots & \ddots & \ddots & \ddots & \ddots & \vdots \\
\mathbf{0} & \ddots & \mathbf{0} & \mathbf{0} & \ddots & \mathbf{0} & \ddots & \ddots & \mathbf{C}_{-L}^{v, v} \\
\mathbf{0} & \ddots & \mathbf{0} & \mathbf{0} & \ddots & \ddots & \mathbf{0} & \ddots & \mathbf{0} \\
\vdots & \ddots & \ddots & \ddots & \ddots & \ddots & \ddots & \ddots & \vdots \\
\mathbf{0} & \ddots & \mathbf{0} & \ddots & \ddots & \ddots & \ddots & \ddots & \mathbf{0} \\
\mathbf{C}_{L}^{v, v} & \ddots & \ddots & \mathbf{0} & \ddots & \ddots & \mathbf{0} & \ddots & \mathbf{0} \\
\vdots & \ddots & \ddots & \ddots & \ddots & \ddots & \ddots & \ddots & \vdots \\
\mathbf{C}_{1}^{v, v} & \cdots & \mathbf{C}_{L}^{v, v} & \mathbf{0} & \cdots & \mathbf{0} & \mathbf{0} & \cdots & \mathbf{0}
\end{array}\right) .
$$

Note that $\mathbf{C}^{v, v}$ is a $2\left(N_{y}-1\right)$-by-2( $\left.N_{y}-1\right)$ block-circulant-circulant-block (BCCB) matrix with $2\left(N_{x}-1\right)$-by-2( $\left.N_{x}-1\right)$ circulant blocks. Let $\mathbf{c}^{v, v}$ be its first column vector and $\hat{\mathbf{c}}^{v, v}$ be the Fourier transform of $\mathbf{c}^{v, v}$

$$
\hat{\mathbf{c}}^{v, v}:=\left(F_{2\left(N_{y}-1\right)} \otimes F_{2\left(N_{x}-1\right)}\right) \mathbf{c}^{v, v} .
$$

Here $F_{2\left(N_{y}-1\right)} \otimes F_{2\left(N_{x}-1\right)}$ represents the two-dimensional Fourier matrix of order $4 N$. It is known that $\mathbf{C}^{v, v}$ has the following diagonalization $[6,15]$,

$$
\mathbf{C}^{v, v}:=\left(F_{2\left(N_{y}-1\right)} \otimes F_{2\left(N_{x}-1\right)}\right)^{-1} \operatorname{diag}\left(\hat{\mathbf{c}}^{v, v}\right)\left(F_{2\left(N_{y}-1\right)} \otimes F_{2\left(N_{x}-1\right)}\right) .
$$

Then we take the following procedures to carry out the fast matrix-vector multiplication $\mathbf{A}_{N}^{v, v} \mathbf{d}_{N}$ for any vector $\mathbf{d}_{N} \in \mathbb{R}^{N}$ :

1. Let the vector $\mathbf{d}_{N}$ be denoted by

$$
\begin{aligned}
\mathbf{d}_{N} & =\left[\mathbf{d}_{(1)}, \mathbf{d}_{(2)}, \ldots, \mathbf{d}_{\left(N_{y}-1\right)}\right]^{T} \\
& =\left[d_{1,1}, \ldots, d_{N_{x}-1,1}, d_{1,2}, \ldots, d_{N_{x}-1,2}, \ldots, d_{1, N_{y}-1}, \ldots, d_{N_{x}-1, N_{y}-1}\right]^{T} .
\end{aligned}
$$

Then expand this vector into a $2 N$-dimensional vector $\mathbf{d}_{2 N}$ by

$$
\mathbf{d}_{2 N}=\left[\mathbf{d}_{(1)}, \mathbf{0}, \mathbf{d}_{(2)}, \mathbf{0}, \ldots, \mathbf{d}_{\left(N_{y}-1\right)}, \mathbf{0}\right]^{T},
$$

where 0 represents a $N_{x}-1$-dimensional zero vector.

2. Define $\mathbf{d}_{4 N}$ by $\mathbf{d}_{4 N}=\left[\mathbf{d}_{2 N}, \mathbf{0}\right]^{T}$, where $\mathbf{0}$ represents a $2 N$-dimensional zero vector. 
3. Compute the two-dimensional fast Fourier transform $\hat{\mathbf{c}}^{v, v}$ by (48) and $\mathbf{w}_{1}=\left(F_{2\left(N_{y}-1\right)} \otimes F_{2\left(N_{x}-1\right)}\right) \mathbf{d}_{4 N}$. Evaluate the Hadamard product $\mathbf{w}_{2}=$ $\hat{\mathbf{c}}^{v, v} \cdot \mathbf{w}_{1}$ and then carry out the two-dimensional inverse fast Fourier transform $\mathbf{w}_{3}=\left(F_{2\left(N_{y}-1\right)} \otimes F_{2\left(N_{x}-1\right)}\right)^{-1} \mathbf{w}_{2}$.

4. Keep only the first $2 N$ entries of the vector $\mathbf{w}_{3}$ to obtain a vector $\mathbf{w}_{4}$ and Remove every other $I-1$ entries of the vector $\mathbf{w}_{4}$ to obtain the vector $\mathbf{A}_{N}^{v, v} \mathbf{d}_{N}$.

We can conclude from the above procedures that the main computational work required for $\mathbf{A}_{N}^{v, v} \mathbf{d}_{N}$ is the computations related to the fast Fourier transform in step 3. It needs $O(4 N \log (4 N))=O(N \log N)$ operations via the fast Fourier transform.

The efficient evaluation and storage of the stiffness matrix and the fast matrix-vector multiplications proved in this section can be applied in the development of any fast Krylov subspace iterative methods. Here, we take the standard conjugate gradient squared (CGS) method for the matrix equation (7) as an example to demonstrate the idea [28, 44].

Note that in the conventional conjugate gradient squared (CGS) method the matrix-vector multiplication requires $O\left(N^{2}\right)$ operations, while other operations can be performed in $O(N)$ operations. Furthermore, the conventional evaluation and storage of the stiffness matrix $\mathbf{A}_{2 N}$ require $O\left(N^{2}\right)$ memory and computations. The efficient mechanism developed in this section reduces the storage and assembly of the stiffness matrix $\mathbf{A}_{2 N}$ to $O(N)$.

We also proved that for any $\mathbf{d}_{N} \in \mathbb{R}^{N}$ the matrix-vector multiplications $\mathbf{A}_{N}^{v, v} \mathbf{d}_{N}, \mathbf{A}_{N}^{v, w} \mathbf{d}_{N}$ and $\mathbf{A}_{N}^{w, w} \mathbf{d}_{N}$ can be evaluated in $O(N \log N)$ operations. Finally, we address the fast matrix-vector multiplication $\mathbf{A}_{2 N} \mathbf{u}_{2 N}$ for any $\mathbf{u}_{2 N} \in \mathbb{R}^{2 N}$ : (i) We first express $\mathbf{u}_{2 N}$ in the form of (8). (ii) We collect odd and even entries of $\mathbf{u}_{2 N}$, respectively, to obtain two $N$-dimensional vectors $\mathbf{v}_{N}$ and $\mathbf{w}_{N}$ as defined in (15). (iii) We apply Theorem 4 to evaluate $\mathbf{A}_{N}^{v, v} \mathbf{v}_{N}$, $\mathbf{A}_{N}^{v, w} \mathbf{w}_{N}, \mathbf{A}_{N}^{w, v} \mathbf{v}_{N}$, and $\mathbf{A}_{N}^{w, w} \mathbf{w}_{N}$ in $O(N \log N)$ operations. (iv) We embed $\mathbf{A}_{N}^{v, v} \mathbf{v}_{N}+\mathbf{A}_{N}^{v, w} \mathbf{w}_{N}$ and $\mathbf{A}_{N}^{w, v} \mathbf{v}_{N}+\mathbf{A}_{N}^{w, w} \mathbf{w}_{N}$ into the odd and even entries of a $2 N$-dimensional vector, respectively, to obtain $\mathbf{A}_{2 N} \mathbf{u}_{2 N}$.

\section{Numerical experiments}

Note that the fast collocation method is merely a traditional collocation method, which significantly reduces the number of entries of the stiffness matrix to be evaluated and assembled as well as the computational complexity 
Table 1: Performance of Gauss, CGS, and the FCGS in Example 1.

\begin{tabular}{|c|c|c|c|c|}
\hline & $N_{x}=N_{y}$ & $\left\|\mathbf{u}_{h}-\mathbf{u}\right\|_{L^{2}}$ & \# of iter. & CPUs \\
\hline \multirow{6}{*}{ Gauss } & $2^{4}$ & $3.2905 \times 10^{-2}$ & & $1.23 \mathrm{~s}$ \\
& $2^{5}$ & $2.3835 \times 10^{-2}$ & & $1 \mathrm{~m} 30 \mathrm{~s}$ \\
& $2^{6}$ & $1.4337 \times 10^{-2}$ & & $16 \mathrm{~h} 25 \mathrm{~m}$ \\
& $2^{7}$ & & out of memory & \\
& $2^{8}$ & & out of memory & \\
& $2^{9}$ & & out of memory & \\
\hline \multirow{5}{*}{ CGS } & & $C_{\alpha}=0.18, \alpha=0.60$ & & \\
& $2^{4}$ & $3.2905 \times 10^{-2}$ & 69 & $0.12 \mathrm{~s}$ \\
& $2^{5}$ & $2.3835 \times 10^{-2}$ & 138 & $2.07 \mathrm{~s}$ \\
& $2^{6}$ & $1.4337 \times 10^{-2}$ & 216 & $50 \mathrm{~s}$ \\
& $2^{7}$ & $7.4180 \times 10^{-3}$ & 338 & $1 \mathrm{~d} 12 \mathrm{~h}$ \\
& $2^{8}$ & & out of memory & \\
& $2^{9}$ & & out of memory & \\
\hline \multirow{5}{*}{ FCGS } & & $C_{\alpha}=0.26, \alpha=0.72$ & & \\
& $2^{4}$ & $3.2905 \times 10^{-2}$ & 73 & $0.44 \mathrm{~s}$ \\
& $2^{5}$ & $2.3835 \times 10^{-2}$ & 138 & $2.3 \mathrm{~s}$ \\
& $2^{6}$ & $1.4337 \times 10^{-2}$ & 225 & $31.8 \mathrm{~s}$ \\
& $2^{7}$ & $7.4180 \times 10^{-3}$ & 340 & $3 \mathrm{~m} 46 \mathrm{~s}$ \\
& $2^{8}$ & $5.7085 \times 10^{-3}$ & 478 & $21 \mathrm{~m} 18 \mathrm{~s}$ \\
& $2^{9}$ & $2.5619 \times 10^{-3}$ & 685 & $3 \mathrm{~h} 42 \mathrm{~m}$ \\
\hline & & $C_{\alpha}=0.28, \alpha=0.73$ & & \\
\hline
\end{tabular}

of the matrix-vector multiplication in any Krylov subspace iterative methods, and so naturally inherits the stability, convergence, and other numerical advantages of traditional collocation methods. In this section we carry out numerical experiments for relatively simple model problems to investigate the improvements of the fast collocation method which is solved by the fast CGS (FCGS) method over the traditional collocation method which is solved with widely used Gaussian elimination (Gauss) and the conventional CGS method. All the solvers are implemented using Matlab and all the numerical experiments are performed on a computer with 8-GB memory.

In the numerical experiments, we consider the peridynamic model (1) 


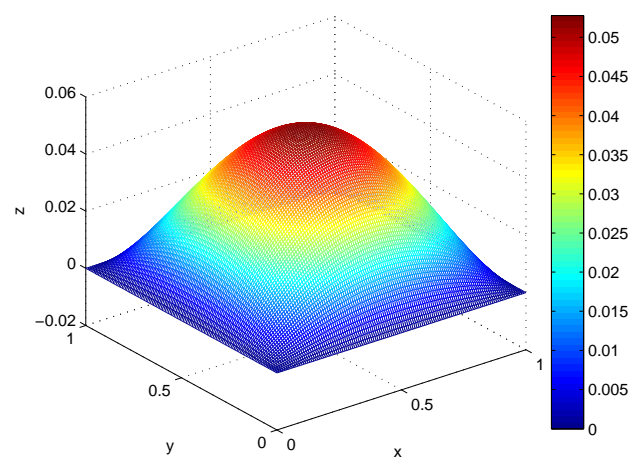

Figure 1: The collocation approximations for $v(x, y)$ (left) and $w(x, y)$ (right) on a $64 \times 64$ mesh in Example 1.

with the kernel function $[34,35]$

$$
\sigma(|(x, y)|)=\frac{1}{\left(x^{2}+y^{2}\right)^{1+s}}, \quad s<1 / 2 .
$$

The domain $\Omega=(0,1) \times(0,1)$ and the neighborhood $B_{\delta}(\mathbf{x})$ is assumed to be a closed disk of radius $\delta=1 / 8$ centered at $\mathbf{x}$, where $\delta$ is refered to as the peridynamic horizon. For simplicity, we choose $h_{x}=h_{y}=h$.
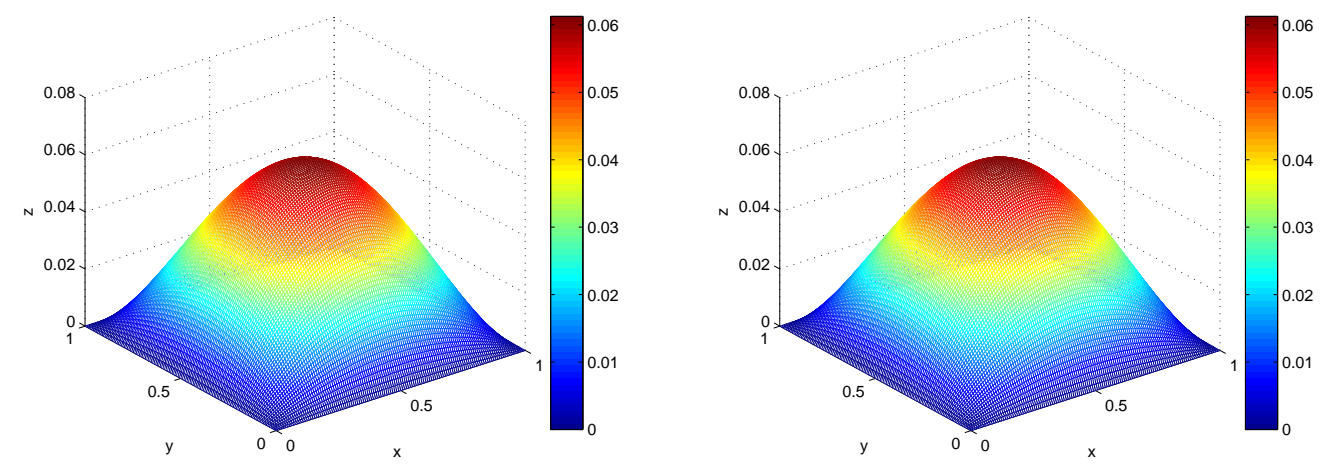

Figure 2: The collocation approximations for $v(x, y)$ (left) and $w(x, y)$ (right) on a $64 \times 64$ mesh in Example 2.

In the numerical experiments we use Gauss numerical quadrature to compute the matrix entries in (11). Furthermore, we use a linear regression to fit 
Table 2: Performance of Gauss, CGS, and FCGS in Example 2.

\begin{tabular}{|c|c|c|c|c|}
\hline & $N_{x}=N_{y}$ & $\left\|\mathbf{u}_{h}-\mathbf{u}\right\|_{L^{2}}$ & \# of iter. & CPUs \\
\hline \multirow{5}{*}{ Gauss } & $2^{4}$ & $1.6387 \times 10^{-2}$ & & $1.22 \mathrm{~s}$ \\
& $2^{5}$ & $6.8624 \times 10^{-3}$ & & $1 \mathrm{~m} 30 \mathrm{~s}$ \\
& $2^{6}$ & $2.3722 \times 10^{-3}$ & & $16 \mathrm{~h} 45 \mathrm{~m}$ \\
& $2^{7}$ & & out of memory & \\
& $2^{8}$ & & out of memory & \\
& $2^{9}$ & & out of memory & \\
\hline \multirow{5}{*}{ CGS } & & $C_{\alpha}=0.81, \alpha=1.39$ & & \\
& $2^{4}$ & $1.6387 \times 10^{-2}$ & 48 & $0.07 \mathrm{~s}$ \\
& $2^{5}$ & $6.8624 \times 10^{-3}$ & 67 & $1.26 \mathrm{~s}$ \\
& $2^{6}$ & $2.3722 \times 10^{-3}$ & 82 & $22.3 \mathrm{~s}$ \\
& $2^{7}$ & $9.4000 \times 10^{-4}$ & 121 & $14 \mathrm{~h} 26 \mathrm{~m}$ \\
& $2^{8}$ & & out of memory & \\
& $2^{9}$ & & out of memory & \\
\hline \multirow{5}{*}{ FCGS } & $2^{4}$ & $1.6387 \times 10^{-2}$ & 48 & $0.42 \mathrm{~s}$ \\
& $2^{5}$ & $6.8624 \times 10^{-3}$ & 71 & $1.31 \mathrm{~s}$ \\
& $2^{6}$ & $2.3722 \times 10^{-3}$ & 87 & $12 \mathrm{~s}$ \\
& $2^{7}$ & $9.4000 \times 10^{-4}$ & 121 & $1 \mathrm{~m} 55 \mathrm{~s}$ \\
& $2^{8}$ & $4.3047 \times 10^{-4}$ & 133 & $12 \mathrm{~m} 3 \mathrm{~s}$ \\
& $2^{9}$ & $2.2423 \times 10^{-4}$ & 151 & $1 \mathrm{~h} 15 \mathrm{~m}$ \\
\hline & & $C_{\alpha}=0.48, \alpha=1.25$ & & \\
\hline
\end{tabular}


the convergence rate $\alpha$ and the associated constant $C_{\alpha}$ in the error estimate

$$
\left\|\mathbf{u}-\mathbf{u}_{h}\right\|_{L^{2}(\Omega)} \leqslant C_{\alpha} h^{\alpha} .
$$

\subsection{A peridynamic model with a continuous displacement}

The exact solution $\mathbf{u}(x, y)=(v(x, y), w(x, y))$ is chosen to be

$$
v(x, y)=w(x, y)=x(1-x) y(1-y), \quad(x, y) \in \Omega
$$

which are also used to define $g^{v}$ and $g^{w}$ on the boundary zone $\Omega_{c}$. The right-hand side external forcing term $\mathbf{b}(x, y)$ can be computed analytically by polar coordinates transformation as follows:

$$
\begin{aligned}
& b^{v}(x, y)=\frac{3 \pi \delta^{2-2 s}}{8-8 s}\left(y-y^{2}\right)+\frac{\pi \delta^{2-2 s}}{8-8 s}\left(3 x+2 y-4 x y-x^{2}-1\right)-\frac{\pi \delta^{4-2 s}}{32-16 s} \\
& b^{w}(x, y)=\frac{3 \pi \delta^{2-2 s}}{8-8 s}\left(x-x^{2}\right)+\frac{\pi \delta^{2}-2 s}{8-8 s}\left(2 x+3 y-4 x y-y^{2}-1\right)-\frac{\pi \delta^{4-2 s}}{32-16 s}
\end{aligned}
$$
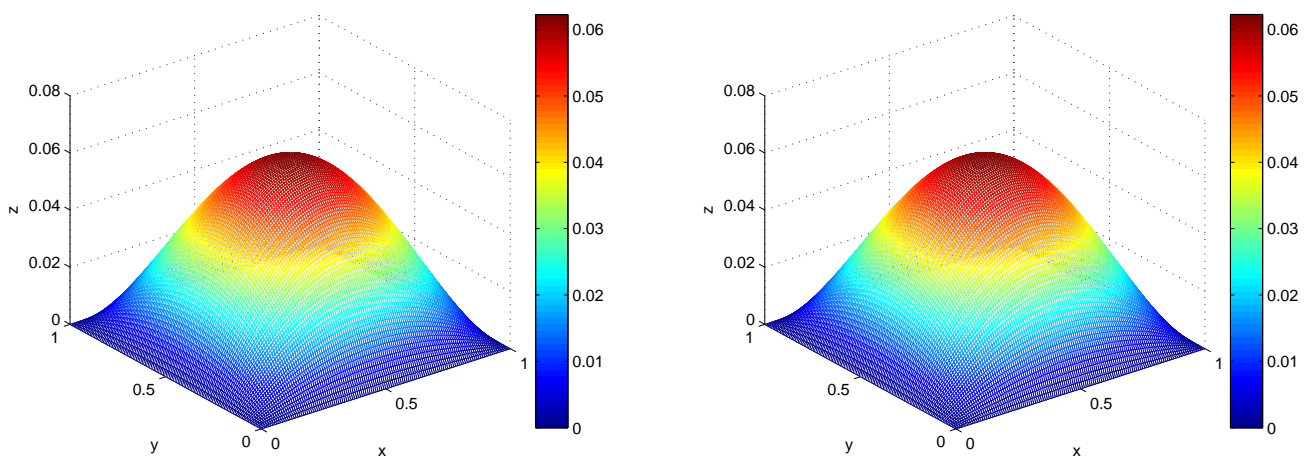

Figure 3: The collocation approximations for $v(x, y)$ (left) and $w(x, y)$ (right) on a $64 \times 64$ mesh in Example 3.

Example 1. We consider the kernel function (50) with $s=3 / 8$. We present the $L^{2}$ errors of the Gauss, CGS, and FCGS numerical solutions, the $\mathrm{CPU}$ time consumed by these methods, and the number of iterations by CGS and FCGS for the mesh sizes from $1 / 2^{4}$ to $1 / 2^{9}$ in Table 1 . We observe that all the three solvers generate identical numerical solutions as we have anticipated, since the fast collocation method is merely the traditional one with a much efficient evaluation and assembly of the stiffness matrix and a 
Table 3: Performance of Gauss, CGS, and FCGS in Example 3.

\begin{tabular}{|c|c|c|c|c|}
\hline & $N_{x}=N_{y}$ & $\left\|\mathbf{u}_{h}-\mathbf{u}\right\|_{L^{2}}$ & \# of iter. & CPUs \\
\hline \multirow{5}{*}{ Gauss } & $2^{4}$ & $9.6360 \times 10^{-3}$ & & $1.23 \mathrm{~s}$ \\
& $2^{5}$ & $3.0229 \times 10^{-3}$ & & $1 \mathrm{~m} 31 \mathrm{~s}$ \\
& $2^{6}$ & $8.2596 \times 10^{-4}$ & & $16 \mathrm{~h} 30 \mathrm{~m}$ \\
& $2^{7}$ & & out of memory & \\
& $2^{8}$ & & out of memory & \\
& $2^{9}$ & & out of memory & \\
\hline \multirow{5}{*}{ CGS } & & $C_{\alpha}=1.34, \alpha=1.77$ & & \\
& $2^{4}$ & $9.6360 \times 10^{-3}$ & 41 & $0.12 \mathrm{~s}$ \\
& $2^{5}$ & $3.0229 \times 10^{-3}$ & 48 & $0.94 \mathrm{~s}$ \\
& $2^{6}$ & $8.2596 \times 10^{-4}$ & 51 & $15.7 \mathrm{~s}$ \\
& $2^{7}$ & $1.9064 \times 10^{-4}$ & 63 & $6 \mathrm{~h} 18 \mathrm{~m}$ \\
& $2^{8}$ & & out of memory & \\
& $2^{9}$ & & out of memory & \\
\hline \multirow{5}{*}{ FCGS } & & $C_{\alpha}=1.93, \alpha=1.89$ & & $0.33 \mathrm{~s}$ \\
& $2^{4}$ & $9.6360 \times 10^{-3}$ & 41 & $0.95 \mathrm{~s}$ \\
& $2^{5}$ & $3.0229 \times 10^{-3}$ & 48 & $4.6 \mathrm{~s}$ \\
& $2^{6}$ & $8.2596 \times 10^{-4}$ & 51 & $5 \mathrm{~m} 1 \mathrm{~s}$ \\
& $2^{7}$ & $1.9064 \times 10^{-4}$ & 63 & $35 \mathrm{~m} 13 \mathrm{~s}$ \\
\hline & $2^{8}$ & $7.2990 \times 10^{-5}$ & 71 & \\
\hline & $2^{9}$ & $2.2827 \times 10^{-5}$ & 80 & \\
\hline & & $C_{\alpha}=1.27, \alpha=1.77$ & & \\
\hline
\end{tabular}


fast evaluation of the matrix-vector multiplication. The numerical results in the table justify that the fast method has a significant improvement of computational complexity over the traditional one. The direct solver uses the most CPU time and runs out of memory for $h=1 / 2^{7}$, while both CGS and FCGS still work for the mesh size $h=1 / 2^{7}, 1 / 2^{8}$, and $1 / 2^{9}$. In fact, $h=1 / 2^{7}$ is the finest mesh size for the CGS to work on this particular computer. However, for $h=1 / 2^{8}$ and $h=1 / 2^{9}$, the FCGS still works because of less memory requirement. In principle, the FCGS still applies to the problem with much finer mesh sizes. The numerical results also show that the FCGS has a significantly reduced CPU time as anticipated. We can also conclude from Table 1 that the convergence rate $\alpha$ of these methods is sublinear.

For clarity, we present a representative figure of the collocation approximations for the displacement field components $v(x, y)$ and $w(x, y)$ on a $64 \times 64$ mesh in Figure 1.

Example 2. We consider the case $s=0$ in (50), which corresponds to a borderline case between a nonintegrable kernel and an integrable kernel. We present the numerical results in Table 2. We virtually have the same observations as in Example 1, except that we observe better accuracy and convergence rates of the numerical solutions and less number of iterations as a reflection of improved condition number of the stiffness matrix. The corresponding numerical solutions for the displacement field components $v(x, y)$ and $w(x, y)$ are shown in Figure 2 .

Example 3. We choose $s=-1 / 2$ in (50). In this case the kernel $\sigma \in L^{1}(\Omega)$ but $\sigma \notin L^{2}(\Omega)$. We present numerical results in Table 3 . We again have the same observations as in Examples 1 and 2, except that a further improved accuracy and a convergence rate of almost 2 of the numerical solutions and further reduced number of iterations. Furthermore, The corresponding numerical solutions for the displacement field components $v(x, y)$ and $w(x, y)$ on a $64 \times 64$ mesh are shown in Figure 3 .

\subsection{A peridynamic model with a discontinuous displacement}

We simulate the peridynamic model (1) with a discontinuous displacement

$$
\begin{aligned}
v(x, y) & =\left\{\begin{array}{cc}
x+y, & x<0.5 \\
x^{2}+y^{2}, & x>0.5
\end{array}\right. \\
w(x, y) & =x(1-x) y(1-y) .
\end{aligned}
$$


Table 4: Performance of Gauss, CGS, and FCGS in Example 4.

\begin{tabular}{|c|c|c|c|c|}
\hline & $N_{x}=N_{y}$ & $\left\|\mathbf{u}_{h}-\mathbf{u}\right\|_{L^{2}}$ & \# of iter. & CPUs \\
\hline \multirow{5}{*}{ Gauss } & $2^{4}$ & $7.2376 \times 10^{-2}$ & & $1.25 \mathrm{~s}$ \\
& $2^{5}$ & $4.1886 \times 10^{-2}$ & & $1 \mathrm{~m} 27 \mathrm{~s}$ \\
& $2^{6}$ & $2.7860 \times 10^{-2}$ & & $17 \mathrm{~h} 5 \mathrm{~m}$ \\
& $2^{7}$ & & out of memory & \\
& $2^{8}$ & & out of memory & \\
& $2^{9}$ & & out of memory & \\
\hline \multirow{5}{*}{ CGS } & & $C_{\alpha}=0.48, \alpha=0.69$ & & \\
& $2^{4}$ & $7.2376 \times 10^{-2}$ & 61 & $0.14 \mathrm{~s}$ \\
& $2^{5}$ & $4.1886 \times 10^{-2}$ & 71 & $1.25 \mathrm{~s}$ \\
& $2^{6}$ & $2.7860 \times 10^{-2}$ & 76 & $20.2 \mathrm{~s}$ \\
& $2^{7}$ & $1.9248 \times 10^{-2}$ & 83 & $8 \mathrm{~h} 51 \mathrm{~m}$ \\
& $2^{8}$ & & out of memory & \\
\hline \multirow{5}{*}{ FCGS } & $2^{9}$ & & out of memory & \\
& & $C_{\alpha}=0.40, \alpha=0.63$ & & $0.53 \mathrm{~s}$ \\
& $2^{4}$ & $7.2376 \times 10^{-2}$ & 61 & $1.22 \mathrm{~s}$ \\
& $2^{5}$ & $4.1886 \times 10^{-2}$ & 71 & $56.4 \mathrm{~s}$ \\
& $2^{6}$ & $2.7860 \times 10^{-2}$ & 76 & $5 \mathrm{~m} 15 \mathrm{~s}$ \\
& $2^{7}$ & $1.9248 \times 10^{-2}$ & 83 & $36 \mathrm{~m} 4 \mathrm{~s}$ \\
\hline & $2^{8}$ & $1.3491 \times 10^{-2}$ & 85 & \\
\hline & $2^{9}$ & $9.5472 \times 10^{-3}$ & 88 & \\
\hline
\end{tabular}



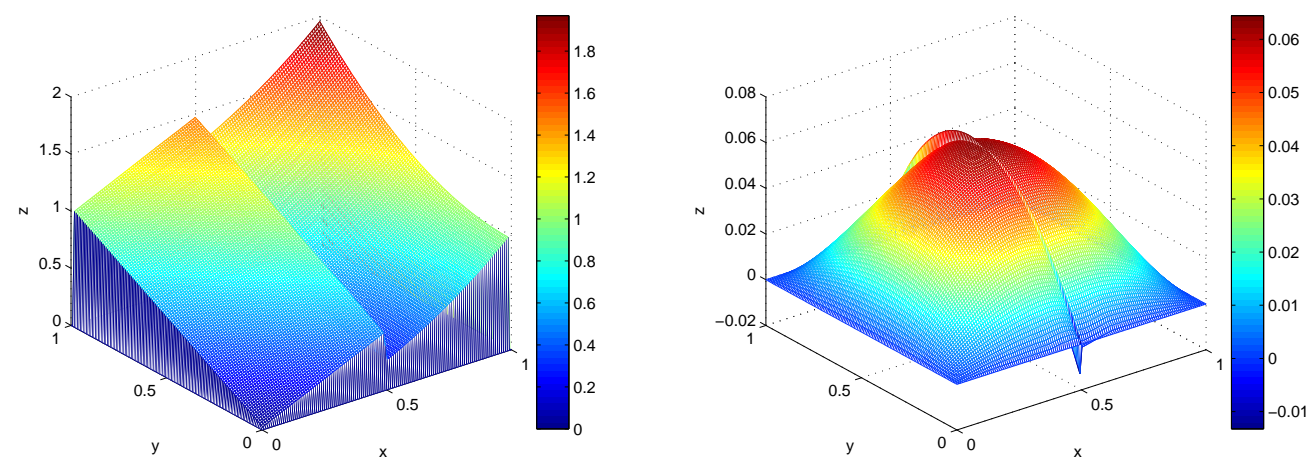

Figure 4: The collocation approximations for $v(x, y)$ (left) and $w(x, y)$ (right) on a $64 \times 64$ mesh in Example 4.

The computational nodes are placed along the discontinuity 0.5. We consider the kernel function (50) with $s=-1 / 2$ as in Example 3. The right-hand side term $\mathbf{b}$ is computed by the numerical integration. We present the numerical results in Table 4. We have similar observations as in Example 3, except that a greatly reduced convergence rate $O\left(h^{1 / 2}\right)$. Because the true solutions are both lack of enough regularity, this convergence rate is the same as the Galerkin finite element method had in [5]. For clarity, we show the numerical solutions for the displacement field components $v(x, y)$ and $w(x, y)$ on a $64 \times$ 64 mesh in Figure 4. From this figure, we can see the numerical solutions at the points where there is a discontinuity have a poor performance.

\section{Conclusions}

In this paper we develop a fast collocation method for a static bondbased linear peridynamic model (1) in two space dimensions. The method reduces the computational work from $O\left(N^{2}\right)$ per Krylov subspace iteration to $O(N \log N)$ and the memory requirement from $O\left(N^{2}\right)$ to $O(N)$. In addition, the method reduces the computational work of evaluating and assembling the stiffness matrix, which often constitutes a major portion of the overall computational work, from $O\left(N^{2}\right)$ to $O(N)$. In fact, the fast collocation method is nothing but the conventional one, and so naturally inherits the stability, convergence, and other numerical advantages of conventional collocation methods [12, 22, 30, 33, 36, 42, 52]. Furthermore, any numerical techniques developed for traditional collocation methods, such as the numerical quadra- 
tures for the accurate evaluation of singular integrals $[2,18,26,27,29,36,51]$, can be applied to the fast collocation method.

In the fast collocation method we take the full advantage of the structure of its stiffness matrix in its storage, evaluation, and assembly and in the efficient solution of the corresponding matrix equation. This would significantly reduce the computational complexity and storage of the fast collocation method over conventional ones, without using any lossy compression. For instance, we would use the same numerical quadratures for conventional methods to evaluate the singular integrals in the stiffness matrix, except that we only need to evaluate $O(N)$ of them instead of $O\left(N^{2}\right)$ of them. Numerical results are presented to show the utility of the fast method.

Technically, the contributions of this paper are summarized as follows: (i) The development of the fast collocation method for the peridynamic model (1) is motivated by that of the fast finite difference methods for FPDEs $[4,24,44,45,49,50]$. However, the peridynamic model (1) gives rise to the coupling in all the directions, in contrast to FPDEs in which the fractional derivatives are expressed only in the coordinate directions, and so represents a much stronger coupling than FPDEs do. In this paper we analyze and utilize the structure of the stiffness matrix to develop a fast collocation method with efficient matrix assembly and storage. (ii) The fast numerical methods developed for FPDEs [44, 45] and nonlocal diffusion models [46, 47] are primarily for scalar problems. However, the peridynamic model (1) is a vector equation. Due to the coupling between the $x$ component $v$ and the $y$ component $w$ of the displacement vector $\mathbf{u}$ introduced by the tensor product kernel in (2), the stiffness matrix in the collocation method is not BTTB as that for nonlocal diffusion models [47]. Thus, the fast numerical methods developed for FPDEs and nonlocal diffusion models do not apply to the peridynamic model (1). In this paper we analyze the coupling effect of the peridynamic model (1) on the stiffness matrix of the collocation method and utilize the structure in the development of a fast collocation method.

We end this section by discussing possible extensions and limitations of the current method: (i) The fast collocation method developed in this paper can be extended to time-dependent problems in a straightforward manner. In fact, the condition number of the stiffness matrix is improved greatly due to the impact of the time step size. (ii) The extension to three-dimensional problem is conceptually straightforward as the stiffness matrix should retain a similar structure, but with one more layer of matrix blocks. However, the practical implementation requires tremendous effort in terms of evaluting 
the three-fold integrals with singular kernels. This will be investigated in the near future. (iii) The extension to state-based peridynamic models[37] is much more involved, as the corresponding peridynamic model doubles the folds of integrals. For example, one needs to calculate a four-fold integral for each entry of the stiffness matrix for a two-dimensional problem. It is not clear whether the stiffness matrix still possesses a somewhat similar structure in this context. This issue will be investigated in the future. (iv) The peridynamic models for problems with material heterogeneities and anisotropy contain a variable-coefficient inside the integral operator [41]. It is not clear whether the idea in the current paper carries over. This issue will be investigated in the future.

\section{Acknowledgements}

The authors would like to express their sincere thanks to Dr. Pablo Seleson for the very helpful discussions about numerical approximations of peridynamic models. The authors would also like to express their sincere thanks to the referees for their very helpful comments and suggestions, which greatly improved the quality and readability of this paper. This work was partially supported by the OSD/ARO MURI Grant W911NF-15-1-0562, by the National Science Foundation under Grants DMS-1216923 and DMS1620194, and the National Natural Science Foundation of China under Grants 91130010, 11471194, and 11571115, and the Taishan research project of Shandong Province.

\section{Appendix}

In this section, we give a detailed description of the fast method by taking an example. We consider a small system by choosing $N_{x}=N_{y}=5, L=$ $K=1$, and $h_{x}=h_{y}=1$ in the discrete system discussed in Section 2. The kernel function $\sigma(x, y)$ is also chosen as (50). In this small system, we have 16 collocation points at which the values of $v(x, y)$ and $w(x, y)$ need to be computed, which are illustrated in Figure 5.

Then we only consider the evaluation and construction of the matrix $\mathbf{T}_{j}^{v, v} \in \mathbb{R}^{16,16}$ for $j=0,-1$, and 1 . The evaluations and constructions of the matrices $\mathbf{T}_{j}^{v, w}$ and $\mathbf{T}_{j}^{w, w}$ are carried out similarly. By the proof of Theorem 2 , the value of each entry in $\mathbf{T}_{j}^{v, v}$ is only determined by the relative position of $\left(x_{i}, y_{j}\right)$ and $\left(x_{i^{\prime}}, y_{j^{\prime}}\right)$, where $1 \leq i, j \leq 4$ and $1 \leq i^{\prime}, j^{\prime} \leq 4$. Thus we can 
choose arbitrary collocation point to compute all the entries in the matrix $\mathbf{T}_{j}^{v, v}$ for $j=0,-1$, and 1 .

Without loss of generality, we choose the point $\left(x_{2}, y_{3}\right)$ as the reference point as depicted in Figure 5. To construct the matrix $\mathbf{T}_{j}^{v, v}$, we need to compute the matrix entries $\mathbf{A}_{m, n}^{v, v}$ defined in (11), where $m$ and $n$ are related to the indices $(i, j)$ and $\left(i^{\prime}, j^{\prime}\right)$ by

$$
\begin{aligned}
& m=4(j-1)+i, \quad i=2, j=3, \\
& n=4\left(j^{\prime}-1\right)+i^{\prime}, \quad 1 \leqslant i^{\prime} \leqslant 3,2 \leqslant j^{\prime} \leqslant 4 .
\end{aligned}
$$

In fact, these entries can be computed analytically. For example, by the transformations $\xi_{1}=x^{\prime}-x_{2}$ and $\xi_{2}=y^{\prime}-y_{3}$, we have

$$
\begin{aligned}
A_{10,15}^{v, v} & =-\int_{\Omega_{1}} \frac{\left(x^{\prime}-x_{2}\right)^{2}}{\left(\left(x^{\prime}-x_{2}\right)^{2}+\left(y^{\prime}-y_{3}\right)^{2}\right)^{2+s}}\left(1+x^{\prime}-x_{3}\right)\left(1+y^{\prime}-y_{4}\right) d x^{\prime} d y^{\prime} \\
& =-\int_{0}^{1} \int_{0}^{\sqrt{1-\xi_{2}^{2}}} \frac{\xi_{1}^{3} \xi_{2}}{\left(\xi_{1}^{2}+\xi_{2}^{2}\right)^{2+s}} d \xi_{1} d \xi_{2} \\
& =-\int_{0}^{1} \int_{0}^{\pi / 2} r^{1-2 s} \cos ^{3} \theta \sin \theta d \theta d r \\
& =-\frac{1}{8-8 s}
\end{aligned}
$$

where $\Omega_{1}=B_{1}\left(x_{2}, y_{3}\right) \cap\left(\left(x_{2}, x_{3}\right) \times\left(y_{3}, y_{4}\right)\right)$ and we have used polar coordinates transformation. Other entries can be computed similarly. 
Let $\alpha:=i^{\prime}-2$ and $\beta:=j^{\prime}-3$ represents the relative position of the points $\left(x_{i}, y_{j}\right)$ and $\left(x_{i^{\prime}}, y_{j^{\prime}}\right)$. Let $t_{\alpha, \beta}^{v, v}=A_{m, n}^{v, v}$, where $m$ and $n$ are related to the indices $(i, j)$ and $\left(i^{\prime}, j^{\prime}\right)$ by $(53)$. Then we can store the 9 nonzero entries by the following matrix of order 3

$$
\mathbf{G}^{v, v}=\left(\begin{array}{ccc}
t_{-1,-1}^{v, v} & t_{-1,0}^{v, v} & t_{-1,1}^{v, v} \\
t_{0,-1}^{v, v} & t_{0,0}^{v, v} & t_{0,1}^{v, v} \\
t_{1,-1}^{v, v} & t_{1,0}^{v, v} & t_{1,1}^{v, v}
\end{array}\right) .
$$

Then we generate the matrix $\mathbf{T}_{j}^{v, v}$ for $j=-1,0$, and 1 using the matrix $\mathbf{G}^{v, v}$ as follows:

$$
\begin{aligned}
\mathbf{T}_{-1}^{v, v}= & \left(\begin{array}{cccc}
t_{0,-1}^{v, v} & t_{1,-1}^{v, v} & 0 & 0 \\
t_{-1,-1}^{v, v} & t_{0,-1}^{v, v} & t_{1,-1}^{v, v} & 0 \\
0 & t_{-1,-1}^{v, v} & t_{0,-1}^{v, v} & t_{1,-1}^{v, v} \\
0 & 0 & t_{-1,-1}^{v, v} & t_{0,-1}^{v, v}
\end{array}\right), \\
\mathbf{T}_{0}^{v, v}= & \left(\begin{array}{cccc}
t_{0,0}^{v, v} & t_{1,0}^{v, v} & 0 & 0 \\
t_{-1,0}^{v, v} & t_{0,0}^{v, v} & t_{1,0}^{v, v} & 0 \\
0 & t_{-1,0}^{v, v} & t_{0,0}^{v, v} & t_{1,0}^{v, v} \\
0 & 0 & t_{-1,0}^{v, v} & t_{0,0}^{v, v}
\end{array}\right), \\
\mathbf{T}_{1}^{v, v}= & \left(\begin{array}{cccc}
t_{0,1}^{v, v} & t_{1,1}^{v, v} & 0 & 0 \\
t_{-1,1}^{v, v} & t_{0,1}^{v, v} & t_{1,1}^{v, v} & 0 \\
0 & t_{-1,1}^{v, v} & t_{0,1}^{v, v} & t_{1,1}^{v, v} \\
0 & 0 & t_{-1,1}^{v, v} & t_{0,1}^{v, v}
\end{array}\right)
\end{aligned}
$$

Then we construct the BTTB matrix $\mathbf{A}_{16}^{v, v}$ by the following way

$$
\mathbf{A}_{16}^{v, v}=\left(\begin{array}{cccc}
\mathbf{T}_{0}^{v, v} & \mathbf{T}_{1}^{v, v} & \mathbf{0} & \mathbf{0} \\
\mathbf{T}_{-1}^{v, v} & \mathbf{T}_{0}^{v, v} & \mathbf{T}_{1}^{v, v} & \mathbf{0} \\
\mathbf{0} & \mathbf{T}_{-1}^{v, v} & \mathbf{T}_{0}^{v, v} & \mathbf{T}_{1}^{v, v} \\
\mathbf{0} & \mathbf{0} & \mathbf{T}_{-1}^{v, v} & \mathbf{T}_{0}^{v, v}
\end{array}\right)
$$

Next we give a detailed description of the matrix-vector multiplication $\mathbf{A}_{N}^{v, v} \mathbf{d}$ for any $\mathbf{d} \in \mathbb{R}^{16}$. It's known that the matrix $\mathbf{T}_{j}^{v, v}$ can be embedded into a $8 \times 8$ circulant matrix $\mathbf{C}_{j}^{v, v}$ for $j=-1,0$, or 1 as follows:

$$
\mathbf{C}_{j}^{v, v}=\left(\begin{array}{ll}
\mathbf{T}_{j}^{v, v} & \tilde{\mathbf{T}}_{j}^{v, v} \\
\tilde{\mathbf{T}}_{j}^{v, v} & \mathbf{T}_{j}^{v, v}
\end{array}\right),
$$


where

$$
\tilde{\mathbf{T}}_{j}^{v, v}=\left(\begin{array}{cccc}
0 & 0 & 0 & t_{-1, j}^{v, v} \\
0 & 0 & 0 & 0 \\
0 & 0 & 0 & 0 \\
t_{1, j}^{v, v} & 0 & 0 & 0
\end{array}\right) .
$$

Then we obtain the BTCB matrix $\mathbf{D}^{v, v}$ by replacing the matrix $\mathbf{T}_{-1}^{v, v}, \mathbf{T}_{0}^{v, v}$, and $\mathbf{T}_{1}^{v, v}$ in the matrix $\mathbf{A}_{16}^{v, v}$ with the circulant matrix $\mathbf{C}_{-1}^{v, v}, \mathbf{C}_{0}^{v, v}$, and $\mathbf{C}_{1}^{v, v}$, respectively, i.e.,

$$
\mathbf{D}^{v, v}=\left(\begin{array}{cccc}
\mathbf{C}_{0}^{v, v} & \mathbf{C}_{1}^{v, v} & \mathbf{0} & \mathbf{0} \\
\mathbf{C}_{-1}^{v, v} & \mathbf{C}_{0}^{v, v} & \mathbf{C}_{1}^{v, v} & \mathbf{0} \\
\mathbf{0} & \mathbf{C}_{-1}^{v, v} & \mathbf{C}_{0}^{v, v} & \mathbf{C}_{1}^{v, v} \\
\mathbf{0} & \mathbf{0} & \mathbf{C}_{-1}^{v, v} & \mathbf{C}_{0}^{v, v}
\end{array}\right)
$$

The matrix $\mathbf{D}^{v, v}$ can also be embedded into a BCCB matrix $\mathbf{C}^{v, v}$ of order 32 as follows:

$$
\mathbf{C}^{v, v}=\left(\begin{array}{ll}
\mathbf{D}^{v, v} & \tilde{\mathbf{D}}^{v, v} \\
\tilde{\mathbf{D}}^{v, v} & \mathbf{D}^{v, v}
\end{array}\right),
$$

where

$$
\tilde{\mathbf{D}}^{v, v}=\left(\begin{array}{cccc}
\mathbf{0} & \mathbf{0} & \mathbf{0} & \mathbf{C}_{-1}^{v, v} \\
\mathbf{0} & \mathbf{0} & \mathbf{0} & \mathbf{0} \\
\mathbf{0} & \mathbf{0} & \mathbf{0} & \mathbf{0} \\
\mathbf{C}_{1}^{v, v} & \mathbf{0} & \mathbf{0} & \mathbf{0}
\end{array}\right)
$$

Let $\mathbf{c}^{v, v}$ be the first column vector of the matrix $\mathbf{C}^{v, v}$. Let $F_{8} \otimes F_{8}$ be the two-dimensional discrete Fourier transform matrix. The Fourier transform of $\mathbf{c}^{v, v}$ is given by

$$
\tilde{\mathbf{c}}^{v, v}=\left(F_{8} \otimes F_{8}\right) \mathbf{c}^{v, v} .
$$

Then the matrix $\mathbf{C}^{v, v}$ has the following diagonalization

$$
\mathbf{C}^{v, v}=\left(F_{8} \otimes F_{8}\right)^{-1} \operatorname{diag}\left(\tilde{\mathbf{c}}^{v, v}\right)\left(F_{8} \otimes F_{8}\right) .
$$

Then we can carry out the fast matrix-vector $\mathbf{A}_{N}^{v, v} \mathbf{d}$ by the procedures in the proof of Theorem 3. Not that we use the Matlab functions fft2 and ifft2 to compute the two-dimensional fast Fourier transform and the twodimensional inverse fast Fourier transform,respectively. 
[1] F. Bobaru and Y.D. Ha, Adaptive refinement and multiscale modeling in 2D peridynamics, Int'l J. Multiscale Comput. Engrg, 9 (2011), 635-659.

[2] F. Bobaru, Y.D. Ha, and W. Hu, Damage progression from impact in layered glass modeled with peridynamics, Central European J. Engrg., 2 (2012) 551-561.

[3] F. Bobaru, M. Yang, L.F. Alves, S.A. Silling, E. Askari, and J. Xu, Convergence, adaptive refinement, and scaling in 1D peridynamics, Int. J. Numer. Meth. Engng., 77 (2009) 852-877.

[4] S. Chen, F. Liu, X. Jiang, I. Turner, and V. Anh, A fast semi-implicit difference method for a nonlinear two-sided space-fractional diffusion equation with variable diffusivity coefficients, Appl. Math. Comp., 257 (2015), 591-601.

[5] X. Chen and M. Gunzburger, Continuous and discontinuous finite element methods for a peridynamics model of mechanics, Comput. Methods Appl. Mech. Engrg., 200 (2011) 1237-1250.

[6] P.J. Davis, Circulant Matrices, Wiley-Intersciences, New York, 1979.

[7] K. Dayal and K. Bhattacharya, Kinetics of phase transformations in the peridynamic formulation of continuum mechanics, J. Mech. Phys. Solids, 54 (2006), 1811-1842.

[8] Q. Du, M. Gunzburger, R. Lehoucq, and K. Zhou, Analysis and approximation of nonlocal diffusion problems with volume constraints, SIAM Rev., 54 (2012), 667-696.

[9] Q. Du, L. Ju, L. Tian, and K.Zhou, A posteriori error analysis of finite element method for linear nonlocal diffusion and peridynamic models, Math. Comput., 82 (2013), 1889-1922.

[10] Q. Du and K. Zhou, Mathematical analysis for the peridynamic nonlocal continuum theory, ESIAM: M2AN Math. Model. Numer. Anal., 45 (2011), $217-234$.

[11] E. Emmrich and O. Weckner, The peridynamic equation and its spatial discretisation, Math. Model. Anal., 12 (2007) 17-27. 
[12] G. Evangelatos and P. Spanos, A collocation approach for spartial discretization of stochastic peridynamic modeling of fracture, J. Mech. Mater. Struct., 6 (2011), 1171-1195.

[13] W. Gerstle, N. Sau, and S. Silling, Peridynamic modeling of concrete structures, Nuclear Engineering and Design, 237 (2007), 1250-1258.

[14] M. Ghajari, L. Iannucci, and P. Curtis, A peridynamic material model for the analysis of dynamic crack propagation in orthotropic media, Comput. Methods Appl. Mech. Engrg., 276 (2014) 431-452.

[15] R.M. Gray, Toeplitz and circulant matrices: a review, Found. Trends Commun., 2 (2006), 155-239.

[16] Y. D. Ha and F. Bobaru, Studies of dynamic crack propagation and crack branching with peridynamics, Int J. Fract., 162 (2010), 229-244.

[17] Y. D. Ha and F. Bobaru, Characteristics of dynamic brittle fracture captured with peridynamics, Engineering Fracture Mechanics, 78 (2011), 1156-1168.

[18] S.F. Henke and S. Shanbhag, Mesh sensitivity in peridynamic simulations, Computer Physics Communications, 185 (2014), 181-193.

[19] W. Hu, Y.D. Ha, and F. Bobaru, Peridynamic model for dynamic fracture in unidirectional fiberreinforced composites, Comput. Methods Appl. Mech. Engrg., 217-220 (2012) 247-261.

[20] B. Kilic, A. Agwai, E. Madenci, Peridynamic theory for progressive damage prediction in centercracked composite laminates, Composite Structures, 90 (2009), 141-151.

[21] B. Kilic and E. Madenci, Prediction of crack paths in a quenched glass plate by using peridynamic theory, Int. J. Fract., 156 (2009), 165-177.

[22] B. Kilic and E. Madenci, Structural stability and failure analysis using peridynamic theory, Int'l J. Non-Linear Mechanics, 44 (2009), 845-854.

[23] X. Lai, B. Ren, H. Fan, S. Li, C. T. Wu, R. A. Regueiro, and L. Liu, Peridynamics simulations of geomaterial fragmentation by impulse loads, Int. J. Numer. Anal. Meth. Geomech, 39 (2015), 1304-1330. 
[24] F. Liu, V. Anh, and I. Turner, Numerical solution of the space fractional Fokker-Planck equation, J. Comput. Appl. Math., 166 (2004), 209-219.

[25] E. Oterkus, E. Madenci, O. Weckner, S. Silling, and P. Bogert, Combined finite element and peridynamic analyses for predicting failure in a stiffened composite curved panel with a central slot, Composite Structures, 94 (2012) 839-850.

[26] M.L. Parks, R.B. Lehoucq, S.J. Plimpton, and S.A. Silling, Implementing peridynamics within a molecular dynamics code, Comp. Phys. Comm., 179 (2008), 777-783.

[27] M.L. Parks, P. Seleson, S.J. Plimpton, S.A. Silling, and R.B. Lehoucq, Peridynamics with LAMMPS: A user guide v0.3 beta, SAND Report 20118523, Sandia National Laboratories, Albuquerque, NM and Livermore, CA (2011).

[28] Y. Saad, Iterative methods for sparse linear systems, 2nd ed., SIAM, Rhode Island, 2003.

[29] P. Seleson, Improved one-point quadrature algorithms for twodimensional peridynamic models based on analytical calculations, Comput. Methods Appl. Mech. Engrg., 282 (2014) 184-217.

[30] P. Seleson, Q. Du, and M. L. Parks, On the consistency between nearestneighbor peridynamic discretizations and discretized classical elasticity models, Submitted.

[31] P. Seleson and D. Littlewood, Convergence studies in meshfree peridynamic simulations, submitted.

[32] P. Seleson and M.L. Parks, On the role of the infuence function in the peridynamic theory, Int'l J. Multiscale Comput. Engrg., 9 (2011), 689-706.

[33] P. Seleson, M.L. Parks, M. Gunzburger, and R.B. Lehoucq, Peridynamics as an upscaling of molecular dynamics, Multiscale Modeling and Simulation, 8 (2009), 204-227.

[34] S. Silling, Reformulation of elasticity theory for discontinuous and longrange forces, J. Mech. Phys. Solids, 48 (2000) 175-209. 
[35] S. Silling, Linearized theory of peridynamic states, J. Elast., 99 (2010) $85-111$.

[36] S. Silling and E. Askari, A meshfree method based on the peridynamic model of solid mechanics, Comput. Struct., 83 (2005) 1526-1535.

[37] S. Silling, M. Epton, O. Wecker, J. Xu, and E. Askari, Peridynamic states and constitutive modeling, J. Elast., 88 (2007) 151-184.

[38] S. Silling and R. Lehoucq, Peridynamic theory of solid mechanics, Adv. Appl. Mech., 44 (2010) 73-168.

[39] S. Silling, O. Weckner, E. Askari, and F. Bobaru, Crack nucleation in a peridynamic solid, Int. J. Fract., 162 (2010), 219-227.

[40] S. Sun and V. Sundararaghavan, A peridynamic implementation of crystal plasticity, Int'l J. Solids and Structures, 51 (2014) 3350-3360.

[41] T. Mengasha and Q. Du, Nonlocal constrained value problems for a linear peridynamic Navier equation, J. Elast., 116 (2014) 27-51.

[42] X. Tian and Q. Du, Analysis and comparison of different approximations to nonlocal diffusion and linear peridynamic equations, SIAM J. Numer. Anal., 51 (2013), 3458-3482.

[43] M.R. Tupek, J.J. Rimoli, and R. Radovitzky, An approach for incorporating classical continuum damage models in state-based peridynamics, Comput. Methods Appl. Mech. Engrg., 263 (2013), 20-26.

[44] H. Wang and T.S. Basu, A fast finite difference method for twodimensional space-fractional diffusion equations, SIAM J. Sci. Comput., 34 (2012), A2444-A2458.

[45] H. Wang, K. Wang, and T. Sircar, A direct $O\left(N \log ^{2} N\right)$ finite difference method for fractional diffusion equations, J. Comput. Phys., 229 (2010), 8095-8104.

[46] H. Wang and H. Tian, A fast Galerkin method with efficient matrix assembly and storage for a peridynamic model, J. Comput. Phys., 231 (2012) 7730-7738. 
[47] H. Wang and H. Tian, A fast and faithful collocation method with efficient matrix assembly for a two-dimensional nonlocal diffusion model, Comput. Methods Appl. Mech. Eng., 273 (2014) 19-36.

[48] J. Xu, A. Askari, O. Weckner, and S. Silling, Peridynamic analysis of impact damage in composite laminates, J. Aerosp. Eng., SPECIAL ISSUE: Impact Mechanics of Composite Materials for Aerospace Application, 21 (2008) 187-194.

[49] Q. Yang, I. Turner, F. Liu and M. Ilis, Novel numerical methods for solving the time-space fractional diffusion equation in 2D, SIAM Sci. Comput., 33 ( 2011), 1159-1180.

[50] Q. Yang, I. Turner, T. Moroney and F. Liu, A finite volume scheme with preconditioned Lanczos method for two-dimensional space-fractional reaction-diffusion equations, Appl. Math. Modelling, 38 (2014), 3755-3762.

[51] K. Yu, X.J. Xin, and K.B. Lease, A new adaptive integration method for the peridynamic theory, Modelling Simul. Mater. Sci. Engrg., 19 (2011) 045003.

[52] X. Zhang, M. Gunzburger, and L. Ju, Nodal-type collocation methods for hypersingular integral equations and nonlocal diffusion problems, Comput. Methods Appl. Mech. Engrg., 299 (2016), 401-420.

[53] K. Zhou and Q. Du, Mathematical and numerical analysis of linear peridynamic models with nonlocal boundary condition, SIAM J. Numer. Anal., 48 (2010), 1759-1780. 